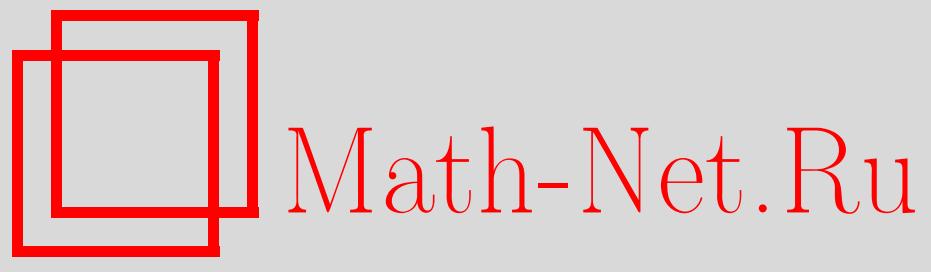

А. Еден, С. В. Зелик, В. К. Калантаров, Контрпримеры к регулярности проекций Мане в теории аттракторов, УМH, 2013, том 68, выпуск 2, 3-32

DOI: https://doi.org/10.4213/rm9512

Использование Общероссийского математического портала Math-Net.Ru подразумевает, что вы прочитали и согласны с пользовательским соглашением http://www . mathnet.ru/rus/agreement

Параметры загрузки:

IP : 3.80 .181 .102

26 апреля 2023 г., 13:18:02

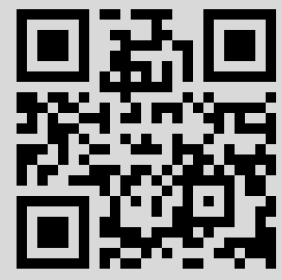




\title{
Контрпримеры к регулярности проекций Мане в теории аттракторов
}

\author{
А. Еден, С. В. Зелик, В. К. Калантаров
}

Работа посвящена изучению глобальных аттракторов абстрактных полулинейных параболических уравнений и их вложений в конечномерные многообразия. Как известно, достаточным условием существования гладких (как минимум, гладкости $C^{1}$ ) инерциальных многообразий конечной размерности, содержащих глобальный аттрактор, является так называемое условие щели в спектре для соответствующего линейного оператора. Также имеется ряд примеров, показывающих, что если щель в спектре отсутствует, то $C^{1}$-гладкого инерциального многообразия может и не быть. $\mathrm{C}$ другой стороны, так как аттрактор обычно имеет конечную фрактальную размерность, то, согласно теореме Мане, он проектируется взаимно однозначно и Гёльдер-гомеоморфно в конечномерную плоскость общего положения, если ее размерность достаточно велика.

В настоящей работе показано, что при отсутствии щели в спектре существуют аттракторы, которые нельзя вложить ни в какое липшицево или даже лог-липшицево конечномерное многообразие. Таким образом, если щель в спектре отсутствует, то в общем случае нельзя ожидать липшицевости или лог-липшицевости обратной проекции Мане аттрактора.

Кроме того, в классе нелинейностей конечной гладкости построены примеры аттракторов с конечной хаусдорфовой и бесконечной фрактальной размерностью.

Библиография: 35 названий.

Ключевые слова: глобальные аттракторы, инерциальные многообразия, проекции Мане, регулярность.

DOI: $10.4213 / \mathrm{rm} 9512$

\section{СОДЕРЖАНИЕ}

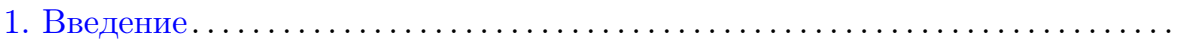

2. Абстрактные параболические уравнения без $C^{1}$-гладких инерциальных

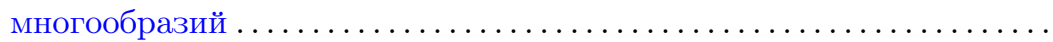

3. Отсутствие липшицевых инерциальных многообразий............. 12

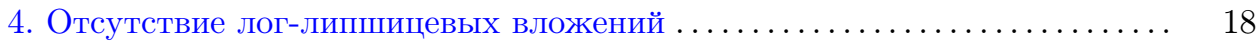

5. "Неправильные" проекции и случай конечной гладкости ............ 24

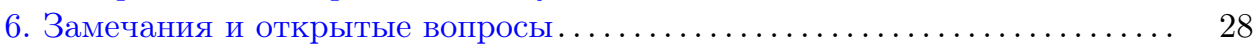

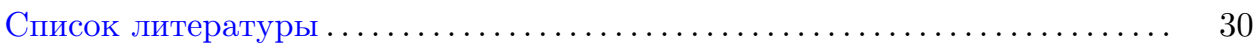

Работа выполнена при поддержке фонда TUBITAK (проект № 107T896).

(C) А. ЕдЕН, С. В. ЗЕЛик, В. К. КАЛАнтАров, 2013 


\section{1. Введение}

Как известно, предельная динамика при больших временах диссипативных систем, порождаемых уравнениями в частных производных (УрЧП), возникающих в математической физике, может быть описана при помощи так называемых глобальных аттракторов. Являясь инвариантным множеством фазового пространства, притягивающим образы всех ограниченных множеств (при $t \rightarrow \infty)$, глобальный аттрактор (если он существует) содержит всю нетривиальную предельную динамику рассматриваемой системы. С другой стороны, аттрактор обычно существенно меньше исходного фазового пространства. В частности, в случае, когда рассматривается УрЧП в ограниченной области $\Omega \subset \mathbb{R}^{n}$, аттрактор $\mathscr{A}$ обычно имеет конечную хаусдорфову и фрактальную размерность (см. [2], [4], [22], [35] и цитируемую там литературу).

Таким образом, несмотря на бесконечномерность исходного фазового пространства (например, $\Phi=L^{2}(\Omega)$ ), предельная динамика оказывается, в некотором смысле, конечномерной и эквивалентной некоторой динамической системе (ДС), определенной на компактном подмножестве $\mathbb{R}^{N}$. Действительно, если фрактальная размерность аттрактора конечна:

$$
\operatorname{dim}_{\mathrm{f}} \mathscr{A}=\kappa<\infty
$$

то, согласно теореме Мане (см. [19]), ортогональный проектор $P$ на плоскость общего положения размерности $N>2 \kappa+1$ является взаимно однозначным на аттракторе $\mathscr{A}$. Поэтому ограничение разрешающей полугруппы $S(t): \Phi \rightarrow \Phi$, $t \geqslant 0$, порожденной рассматриваемой диссипативной системой, на глобальный аттрактор $\mathscr{A}$ гомеоморфно полугруппе $\widetilde{S}(t)$, действующей на конечномерном множестве $P \mathscr{A} \subset \mathbb{R}^{N}$ :

$$
\widetilde{S}(t): P \mathscr{A} \rightarrow P \mathscr{A}, \quad \widetilde{S}(t) v:=P S(t) P^{-1} v, \quad v \in P \mathscr{A} .
$$

Таким образом, по крайней мере с точки зрения топологической динамики нет существенной разницы между бесконечномерными диссипативными системами (глобальный аттрактор которых конечномерен) и классическими конечномерными ДС (см., например, [13] и цитируемую там литературу).

Заметим, однако, что теорема Мане гарантирует только непрерывность редуцированной ДС $\widetilde{S}(t)$, чего недостаточно для более детального ее исследования, так как наиболее эффективные методы современной теории ДС требуют, чтобы рассматриваемая система была гладкой (см., например, [14]). Поэтому фактическая гладкость ДС (1.2) является ключевым вопросом, определяющим эффективность описанной выше конечномерной редукции. Так как исходная бесконечномерная ДС $S(t)$ обычно является гладкой, представляется естественным доказывать регулярность редуцированной ДС $\widetilde{S}(t)$, проверяя дополнительную регулярность обратного проектора Мане $P^{-1}$ на редуцированном аттракторе $P \mathscr{A} \subset \mathbb{R}^{N}$. Таким образом, исследование гладкости обратного проектора Мане становится одной из центральных проблем рассматриваемой теории (см. [29] и цитируемую там литературу). 
Идеальная ситуация возникает в случае, когда рассматриваемая диссипативная система допускает так называемое инерииальное многообразие. Это конечномерное инвариантное подмногообразие $\mathscr{M}$ фазового пространства, диффеоморфное $\mathbb{R}^{N}$, содержащее глобальный аттрактор $\mathscr{A}$ и обладающее свойством асимптотической полноты (т. е. любая траектория исходной ДС экспоненциально притягивается к некоторой траектории, лежащей на многообразии, когда время стремится к бесконечности, см. [8], [9], [20], [35] и цитируемую там литературу). В этом случае предельная динамика описывается редуцированной системой обыкновенных дифференциальных уравнений (ОДУ) на многообразии $\mathscr{M}=\mathbb{R}^{N}$ :

$$
y^{\prime}(t)=\mathscr{F}(y(t)), \quad y \in \mathbb{R}^{N},
$$

которая называется инерииальной формой исходной ДС, а гладкость векторного поля $\mathscr{F}$ определяется фактической гладкостью многообразия $\mathscr{M}$ (см. [6]).

Однако, являясь частным случаем центрального многообразия, инерциальное многообразие требует наличия разложения фазового пространства на "быстрые" и "медленные" переменные, а это, в свою очередь, приводит к весьма ограничительному условию щели в спектре соответствующего линейного оператора.

Действительно, рассмотрим следующее абстрактное полулинейное параболическое уравнение:

$$
\partial_{t} u+A u=F(u),\left.\quad u\right|_{t=0}=u_{0} \in H,
$$

где $A$ - самосопряженный положительно определенный линейный оператор в гильбертовом пространстве $H$, обратный к которому компактен. Пусть $0<$ $\lambda_{1} \leqslant \lambda_{2} \leqslant \cdots$ - собственные значения этого оператора, упорядоченные в порядке возрастания, и пусть $F: H \rightarrow H$ - гладкое и глобально липшицево отображение в $H$ с константой Липшица $L$ (для простоты мы будем рассматривать только случай, когда нелинейный оператор не понижает гладкость, хотя все сказанное ниже может быть обобщено - с минимальными изменениями - на общий случай, когда $F$ понижает гладкость, но подчинен линейному оператору $A)$. В этом случае условие щели в спектре, которое гарантирует существование инерциального многообразия размерности $N$, имеет следующий вид:

$$
\lambda_{N+1}-\lambda_{N}>2 L
$$

(см. [10], [20], [30] и цитируемую там литературу). В частности, даже для уравнений реакции-диффузии условие щели в спектре выполнено, вообще говоря, только в случае одной пространственной переменной (см. [17] по поводу существования инерциального многообразия для некоторых уравнений в случае пространственной размерности два и три при отсутствии щели в спектре). Отметим также, что даже в этом идеальном случае щель в спектре недостаточно велика, чтобы гарантировать $C^{2}$-гладкость многообразия, т. е. даже при наличии щели в спектре, вообще говоря, можно рассчитывать только на $C^{1+\varepsilon}$-гладкость многообразия $\mathscr{M}$ для некоторого малого положительного $\varepsilon$. В общем случае, как известно, если щель в спектре отсутствует, то гладкого инерциального многообразия может и не быть, а глобальный аттрактор может не вкладываться ни 
в какое конечномерное $C^{1}$-многообразие (см. контрпримеры в [18], [31], а также в разделе 2 ниже).

Таким образом, при отсутствии щели в спектре (и при отсутствии инерциального многообразия) нет оснований ожидать, что редуцированная система (1.2) будет $C^{1}$-гладкой. С другой стороны, усиленная версия теоремы Мане (см. [3], [7], [12], [23]) гарантирует, что обратный проектор $P^{-1}$ является непрерывным по Гёльдеру на $P \mathscr{A}$ с константой Гёльдера $\alpha<1$ (для $N$-мерных плоскостей общего положения при условии $N>2 \kappa+1)$. Поэтому редуцированная ДС (1.2) также оказывается непрерывной по Гёлъдеру, и, при некоторых естественных дополнительных условиях на исходную диссипативную систему, константа Гёльдера $\alpha$ может быть сделана сколь угодно близкой к единице за счет увеличения размерности $N$ (см. [12], [26], [29] и цитируемую там литературу). Также известно, что редуцированная ДС $\widetilde{S}(t)$ может быть описана системой ОДУ $(1.3)$ в $\mathbb{R}^{N}$ для некоторого непрерывного по Гёльдеру на $P \mathscr{A}$ векторного поля $\mathscr{F}$ (см. [6]).

Необходимо отметить, однако, что описанное выше представление редуцированной ДС в виде системы ОДУ обладает еще одним существенным недостатком. Действительно, так как непрерывности по Гёльдеру векторного поля Я્F недостаточно для единственности решения, редуцированная система (1.3) не позволяет, вообще говоря, восстановить полугруппу (1.2), поэтому приходится использовать исходную нередуцированную диссипативную систему для выделения "правильного" решения редуцированной системы (1.3). Эта проблема была бы решена, если бы удалось показать, что обратный проектор Мане обладает большей регулярностью, например, является липшицевым, что не противоречит упомянутым выше контрпримерам к $C^{1}$-регулярности и гарантирует единственность решения для редуцированной системы ОДУ (1.3).

Проблемы, связанные с дополнительной регулярностью проекторов Мане (большей, чем непрерывность по Гёльдеру), изучались и продолжают активно изучаться многими авторами (см. [24], [25], [27], [29] и цитируемую там литературу). В частности, ряд необходимых и достаточных условий для липшищевости $P^{-1}$ и векторного поля $\mathscr{F}$ в случае абстрактного полулинейного уравнения (1.4) был получен А. В. Романовым (см. [32], [33]). Например, проектор Мане с липшицевым обратным существует тогда и только тогда, когда сужение оператора $A$ на аттрактор является липшицевым в метрике пространства $H$ :

$$
\left\|A u_{1}-A u_{2}\right\|_{H} \leqslant C\left\|u_{1}-u_{2}\right\|_{H} \quad \forall u_{1}, u_{2} \in \mathscr{A} .
$$

Эта теория, к примеру, позволила проверить существование липшицевых проекторов Мане для следующего класса одномерных уравнений реакции-диффузии:

$$
u_{t}=u_{x x}-f\left(u, u_{x}\right),
$$

для которого условие щели в спектре не выполняется (из-за наличия зависимости от $u_{x}$ в нелинейности) и для которого до сих пор ничего не известно о существовании инерциального многообразия.

Необходимо отметить, однако, что проверка условия (1.6) для более общих классов уравнений является очень сложной задачей и к настоящему времени не 
разработано никаких эффективных методов для этой проверки. С другой стороны, используя стандартные оценки для обратной единственности решений параболических уравнений, основанные на свойстве логарифмической выпуклости, можно получить следующую ослабленную версию неравенства (1.6):

$$
\left\|A u_{1}-A u_{2}\right\|_{H} \leqslant C\left\|u_{1}-u_{2}\right\|_{H} \log \frac{C}{\left\|u_{1}-u_{2}\right\|_{H}} \quad \forall u_{1}, u_{2} \in \mathscr{A},
$$

см. [1], [16] (фактически, в нашем случае, когда $F \in C^{1}(H, H)$, неравенство (1.8) можно усилить, заменив логарифм в правой части квадратным корнем из логарифма, см. [34]). По этой причине выглядит естественным ослабить "непроверяемое" условие липшицевости проектора Мане и попытаться проверить вместо этого его лог-липшицевость (см. [27], [28]). Действительно, как нетрудно видеть, логарифмической липшицевости обратного проектора Мане (в пространстве $\left.H^{2}:=D(A)\right)$ в форме

$$
\left\|P^{-1}\left(v_{1}-v_{2}\right)\right\|_{H^{2}} \leqslant C\left\|v_{1}-v_{2}\right\|_{H^{2}} \log \frac{C}{\left\|v_{1}-v_{2}\right\|_{H^{2}}} \quad \forall v_{1}, v_{2} \in P \mathscr{A} \subset \mathbb{R}^{N}
$$

( $H^{2}$-нормы в правой части можно заменить на соответствующие $H$-нормы, так как все нормы в конечномерном пространстве эквивалентны) достаточно для проверки аналогичной лог-липшицевости векторного поля $\mathscr{F}$ в уравнениях (1.3), что, в свою очередь, влечет единственность решения.

Необходимо также отметить, что несмотря на внешнюю привлекательность идеи о лог-липшицевости обратного проектора Мане и наличие ряда работ, посвященных исследованию условий, ее гарантирующих (главным образом, для случая абстрактных множеств в бесконечномерных пространствах, не обязательно являющихся аттракторами, см. [28] и цитируемую там литературу), нам не известно ни одного примера конкретного уравнения математической физики (или даже какого-либо естественного примера абстрактного параболического уравнения вида (1.4)), где бы эта идея позволила продвинуться дальше теории Романова. С другой стороны, насколько нам известно, ни одного контрпримера к липшицевости или лог-липшицевости обратного проектора Мане для аттракторов диссипативных УрЧП или абстрактных параболических уравнений также не было построено.

Основной целью данной работы как раз и является построение таких контрпримеров в классе абстрактных полулинейных параболических уравнений (1.4). В частности, будет показано, что при отсутствии щели в спектре нельзя ожидать наличия лог-липшицевости обратного проектора Мане, по крайней мере для случая аттракторов уравнений вида (1.4). Точнее, будет доказана следующая теорема, которая является основным результатом работы.

Tеорема 1.1. Пусть $L_{0}:=\sup _{n \in \mathbb{N}}\left(\lambda_{n+1}-\lambda_{n}\right)<\infty$. Тогда для любого положительного L такого, что

$$
L>\max \left\{\frac{L_{0}}{2}, \lambda_{2}\right\}
$$


существует $C^{\infty}$-гладкое нелинейное отображение $F: H \rightarrow H$ с глобальной липшицевой константой $L$ такое, что задача (1.4) имеет компактный глобальный аттрактор $\mathscr{A}$ и этот аттрактор не может быть вложен ни в какое конечномерное лог-липшицево многообразие, в частности, не существует никакой проекиии Мане этого аттрактора с лог-липшицевым обратным оператором.

Отметим, что наша конструкция нелинейности $F$ базируется на классических контрпримерах к теории Флоке для абстрактных линейных параболических уравнений с периодическими коэффициентами (см. [5], [15], [21]). Мы будем использовать такое линейное уравнение, не допускающее ни одной экспоненты Флоке, для вложения "почти" ортогональных последовательностей в соответствующий глобальный аттрактор (см. разделы 3 и 4).

Статья организована следующим образом.

В разделе 2 мы приведем слегка обобщенный вариант контрпримера Романова, показывающий отсутствие $C^{1}$-гладкого инерциального многообразия при нарушении условия щели в спектре. Хотя этот результат представляется известным, нам не удалось найти точную ссылку, поэтому, ввиду важности этого результата для дальнейшего, мы приводим подробное его изложение здесь.

В разделе 3 мы покажем, что отсутствие щели в спектре оператора $A$ позволяет построить периодический по времени линейный оператор $\Phi(t)$ так, чтобы все решения абстрактного параболического уравнения

$$
\partial_{t} v+A v=\Phi(t) v
$$

убывали быстрее чем экспоненциально (как $e^{-t^{2}}$ ) при $t \rightarrow \infty$. Используя этот результат, мы построим затем нелинейность $F$ для уравнения (1.4) таким образом, чтобы нашлись две траектории $u_{1}(t)$ и $u_{2}(t)$, лежащие на аттракторе, такие, что

$$
\left\|u_{1}(t)-u_{2}(t)\right\|_{H} \leqslant C e^{-\alpha t^{2}}, \quad t \geqslant 0 .
$$

Согласно критерию Романова (см. [32]), это исключает возможность существования липшицева инерциального многообразия, так же как и существование проекций Мане с липшицевым обратным оператором.

Раздел 4 посвящен доказательству главной теоремы 1.1. Сначала мы определим так называемый логарифмический фактор удвоения (обобщающий фактор удвоения и размерность Булигана (Bouligand), см. [24]), который должен быть конечным, если аттрактор может быть вложен в конечномерное лог-липшицево многообразие. Затем, при помощи правильно подобранных малых возмущений уравнения, построенного в предыдущем разделе, в различных ортогональных направлениях, будет построено вложение "почти ортогональной" последовательности с бесконечным логарифмическим фактором удвоения в аттрактор соответствующего параболического уравнения. Это исключает возможность существования проекций Мане с лог-липшицевым обратным оператором и показывает, что в общем случае при отсутствии инерциального многообразия от обратной проекции Мане на аттракторе можно ожидать только непрерывности по Гёльдеру. 
В разделе 5 мы построим еще два контрпримера, тесно связанных с контрпримерами предыдущих разделов. Один из них показывает, что в случае конечной гладкости нелинейности $F$ фрактальная размерность аттрактора может зависеть от выбора фазового пространства $H^{s}:=D\left(A^{s / 2}\right)$. В частности, будет предъявлен пример аттрактора с бесконечной фрактальной и конечной хаусдорфовой размерностью (в некотором $H^{s}$ ).

Второй, потенциально более интересный пример иллюстрирует тот факт, что, в отличие от хаусдорфовой или фрактальной размерности, логарифмический фактор удвоения (как и размерность Булигана) может существенно увеличиваться при проектировании на "неправильную" плоскость. А именно, будет построен пример аттрактора, лежащего в двумерном гладком инерциальном многообразии, такого, что его образ $Q_{2} \mathscr{A}$ при ортогональной проекции на плоскость коразмерности два имеет бесконечный логарифмический фактор удвоения и, следовательно, не может быть вложен ни в какое конечномерное лог-липшицево многообразие. В частности, этот пример позволяет сформулировать следующий интересный вопрос.

Пусть аттрактор $\mathscr{A}$ не допускает лог-липшицевых проекций Мане. Можно ли найти "поднятие" $\overline{\mathscr{A}} \subset \bar{H}$ аттрактора $\mathscr{A}$ (или/и ДС (1.4)) в большее гильбертово пространство $\bar{H}$ так, чтобы $\mathscr{A}$ был проекцией $\overline{\mathscr{A}}$ в $H$ и больший аттрактор $\overline{\mathscr{A}}$ допускал более регулярные проекции Мане?

Положительный ответ на этот вопрос в общем случае позволил бы решить проблему с отсутствием лог-липшицевых проекций Мане.

Наконец, в разделе 6 мы обсудим некоторые следствия полученных результатов, а также связанные с ними открытые проблемы.

\section{2. Абстрактные параболические уравнения без $C^{1}$-гладких инерциальных многообразий}

В этом разделе мы покажем, слегка модифицировав контрпример из [32], что условие щели в спектре является, вообще говоря, и необходимым для существования инерциального многообразия в следующем классе абстрактных полулинейных параболических уравнений:

$$
\partial_{t} u+A u=F(u),\left.\quad u\right|_{t=0}=u_{0} \in H .
$$

Здесь $H$ - гильбертово пространство, $A: D(A) \rightarrow H$ - положительный самосопряженный линейный оператор в $H$ с компактным обратным, а $F$ - нелинейный оператор, удовлетворяющий некоторым естественным условиям, сформулированным ниже. Пусть также $\lambda_{1} \leqslant \lambda_{2} \leqslant \cdots \leqslant \lambda_{n} \leqslant \cdots-$ собственные значения оператора $A$, упорядоченные в порядке возрастания, а $\left\{e_{n}\right\}_{n=1}^{\infty}$ - соответствующие им собственные векторы.

Основным результатом этого раздела можно считать следующую теорему.

Теорема 2.1. Пусть собственные значения оператора А удовлетворяют условию

$$
L_{0}:=\sup _{n \in \mathbb{N}}\left(\lambda_{n+1}-\lambda_{n}\right)<\infty
$$


Тогда для любого $L>\max \left\{L_{0} / 2, \lambda_{1}\right\}$ существует гладкий нелинейный операmор $F \in C^{\infty}(H, H)$ такой, что

1) $F$ является глобально липшицевым на $H$ с константой Липшица $L$ :

$$
\|F(u)-F(v)\|_{H} \leqslant L\|u-v\|_{H} \quad \forall u, v \in H,
$$

2) задача (2.1) обладает компактным глобальным аттрактором $\mathscr{A}$ в $H$,

3 ) не существует конечномерного инвариантного $C^{1}$-подмногообразия в $H$, содержащего аттрактор $\mathscr{A}$.

ДокАЗАТЕЛЬСтво. Для построения этого контрпримера нам понадобится следующая простая лемма.

ЛЕмма 2.2. Рассмотрим следующую двумерную систему линейных ОДУ:

$$
\left\{\begin{array}{l}
\frac{d}{d t} u_{n}+\lambda_{n} u_{n}=L u_{n+1}, \\
\frac{d}{d t} u_{n+1}+\lambda_{n+1} u_{n+1}=-L u_{n}
\end{array}\right.
$$

Тогда, если $2 L>\lambda_{n+1}-\lambda_{n}$, то соответствующее характеристическое уравнение не имеет вещественных корней.

ДокАзАтЕльство. Действительно, характеристическое уравнение имеет вид

$$
\operatorname{det}\left(\begin{array}{cc}
-\lambda_{n}-\lambda & L \\
-L & -\lambda_{n+1}-\lambda
\end{array}\right)=0, \quad \lambda^{2}+\left(\lambda_{n}+\lambda_{n+1}\right) \lambda+\lambda_{n} \lambda_{n+1}+L^{2}=0,
$$

а его корни находятся по формуле $\lambda=\alpha_{n} \pm i \omega_{n}$, где

$$
\alpha_{n}:=-\frac{\lambda_{n}+\lambda_{n+1}}{2}, \quad \omega_{n}:=\frac{1}{2} \sqrt{4 L^{2}-\left(\lambda_{n+1}-\lambda_{n}\right)^{2}}>0,
$$

и лемма доказана.

Теперь мы готовы построить искомую нелинейность для уравнения (2.1). Согласно [32], достаточно найти два положения равновесия $u_{+}$и $u_{-}$уравнения (2.1) таких, что спектр $\sigma\left(-A+F^{\prime}\left(u_{-}\right)\right)$не содержит вещественных собственных значений, а спектр $\sigma\left(-A+F^{\prime}\left(u_{+}\right)\right)$содержит в точности одно вещественное и положительное собственное значение (а все остальные собственные значения разбиваются на пары комплексно-сопряженных).

Действительно, предположим, что $C^{1}$-гладкое инвариантное многообразие $\mathscr{M}$ существует и $\operatorname{dim} \mathscr{M}=n$. Тогда из инвариантности следует, что

$$
\sigma_{\mathscr{M}}\left(u_{ \pm}\right):=\sigma\left(\left.\left(-A+F^{\prime}\left(u_{ \pm}\right)\right)\right|_{\mathscr{T} \mathscr{M}\left(u_{ \pm}\right)}\right) \subset \sigma\left(-A+F^{\prime}\left(u_{ \pm}\right)\right),
$$

где $\mathscr{T} \mathscr{M}\left(u_{ \pm}\right)$- касательные плоскости к $\mathscr{M}$ в точках $u=u_{ \pm}$(принадлежащих многообразию, так как все положения равновесия принадлежат аттрактору, а наше многообразие его содержит). В частности, так как наше уравнение вещественное, а все собственные значения из $\sigma_{\mathscr{M}}\left(u_{-}\right)$разбиты на пары комплексно-сопряженных, мы видим, что размерность $\operatorname{dim} \mathscr{M}$ должна быть 
четной (если $\lambda \in \sigma_{\mathscr{M}}\left(u_{-}\right)$, то и $\bar{\lambda} \in \sigma_{\mathscr{M}}\left(u_{-}\right)$, и $\lambda \neq \bar{\lambda}$, так как вещественных собственных значений нет).

Рассмотрим теперь положение равновесия $u_{+}$. Здесь мы имеем в точности одно вещественное собственное значение, которое должно принадлежать $\sigma_{\mathscr{M}}\left(u_{+}\right)$, так как неустойчивое многообразие положения равновесия $u_{+}$лежит в аттракторе. Так как все остальные собственные значения разбиты на пары комплексно-сопряженных, то, аналогично предыдущему случаю, можно сделать вывод, что размерность $\mathscr{M}$ должна быть нечетной. Данное противоречие показывает, что конечномерного $C^{1}$-гладкого инвариантного многообразия действительно не существует.

Таким образом, остается построить нелинейность $F$ так, чтобы соответствующее параболическое уравнение обладало положениями равновесия с описанными выше свойствами. Для этого зафиксируем $u_{ \pm}= \pm N e_{1}$, где $N$ достаточно большое число, и определим отображения $F^{ \pm}(u)$ покоординатно, $F_{n}^{ \pm}(u):=\left(F(u), e_{n}\right), u=\sum_{n=1}^{\infty} u_{n} e_{n}$, в ортонормированном базисе $\left\{e_{n}\right\}$ по следующим формулам:

$$
\begin{aligned}
& F_{1}^{-}(u):=-\lambda_{1} N+L u_{2}, \quad F_{2}^{-}(u):=-L\left(u_{1}+N\right), \\
& F_{2 n+1}^{-}(u):=L u_{2 n+2}, \quad F_{2 n+2}^{-}(u):=-L u_{2 n+1}, \quad n \geqslant 1 \text {, }
\end{aligned}
$$

и

$$
\begin{gathered}
F_{1}^{+}(u):=\lambda_{1} N+L\left(u_{1}-N\right), \\
F_{2 n}^{+}(u):=-L u_{2 n+1}, \quad F_{2 n+1}^{+}(u):=L u_{2 n}, \quad n \geqslant 1 .
\end{gathered}
$$

Оба отображения $F^{-}$и $F^{+}$являются гладкими и глобально липшицевыми с константой Липшица $L$. Кроме того, можно построить гладкое нелинейное отображение $F(u)$ так, чтобы выполнялось соотношение

$$
F(u) \equiv F^{ \pm}(u), \quad \text { если } u \text { близко к } u_{ \pm},
$$

и глобальная константа Липшица оператора $F$ была меньше чем $L+\varepsilon$ (где $\varepsilon=\varepsilon(N)$ может быть сделано сколь угодно малым за счет увеличения $N$, см. следующий раздел по поводу явной конструкции этого отображения). Наконец, можно обрезать нелинейность $F$ вне достаточно большого шара, сделав ее диссипативной, не увеличивая при этом константу Липшица (см. [35] по поводу деталей). Это гарантирует существование компактного глобального аттрактора $\mathscr{A}$.

Вычислим теперь $\sigma\left(-A+F^{\prime}\left(u_{ \pm}\right)\right)$на положениях равновесия $u_{ \pm}$. Действительно, согласно конструкции $F$, линеаризация уравнения $(2.1)$ на положении равновесия $u=u_{-}$имеет вид

$$
\frac{d}{d t} v_{2 n-1}=-\lambda_{2 n-1} v_{2 n-1}+L v_{2 n}, \quad \frac{d}{d t} v_{2 n}=-\lambda_{2 n} v_{2 n}-L v_{2 n-1}, \quad n=1,2, \ldots,
$$

и, благодаря условию $L>L_{0} / 2$, вещественных собственных значений в спектре $\sigma\left(-A+F^{\prime}\left(u_{ \pm}\right)\right)$нет (см. лемму 2.2). 
В отличие от этого, линеаризация на положении равновесия $u=u_{+}$имеет вид

$$
\begin{aligned}
\frac{d}{d t} v_{1} & =\left(L-\lambda_{1}\right) v_{1} \\
\frac{d}{d t} v_{2 n} & =-\lambda_{2 n} v_{2 n}+L v_{2 n+1}, \quad \frac{d}{d t} v_{2 n+1}=-\lambda_{2 n+1} v_{2 n+1}-L v_{2 n}, \quad n=1,2, \ldots .
\end{aligned}
$$

Таким образом, так как $L>\lambda_{1}$, мы имеем в точности одно вещественное и положительное собственное значение, а все остальные являются комплексно-сопряженными по лемме 2.2. Это показывает отсутствие конечномерного $C^{1}$-гладкого инвариантного многообразия и завершает доказательство теоремы.

ЗАмечАниЕ 2.3. Заметим, что $L<L_{0} / 2$ - это классическое условие щели в спектре, которое гарантирует наличие конечномерного инерциального многообразия для уравнения (2.1), см. [20], [30]. Дополнительное условие $L>\lambda_{1}$ необходимо для того, чтобы иметь неустойчивость в уравнении $(2.1)$, в противном случае нелинейность будет недостаточно сильной, чтобы компенсировать диссипативность оператора $A$, и аттрактор будет состоять из одной экспоненциально устойчивой точки (которую естественно считать нульмерным инерциальным многообразием рассматриваемой задачи). Таким образом, доказанная теорема, действительно, показывает неулучшаемость условия щели в спектре для случая абстрактных параболических уравнений и $C^{1}$-гладких инерциальных многообразий.

\section{3. Отсутствие липшицевых инерциальных многообразий}

В этом разделе мы усовершенствуем контрпример из предыдущего раздела, показав при этом, что при отсутствии щели в спектре может не быть не только $C^{1}$-гладкого, но и липшицева инвариантного многообразия, содержащего аттрактор. Точнее, будут предъявлены две траектории $u$ и $v$, лежащие на аттракторе, такие, что

$$
\|u(t)-v(t)\|_{H} \leqslant C e^{-\kappa t^{2}}, \quad t \geqslant 0,
$$

для некоторых положительных $C$ и $\kappa$. Отсюда будет следовать, что аттрактор не может быть спроектирован взаимно однозначно ни на какую конечномерную плоскость так, чтобы прямой и обратный проекторы были липшицевыми, а также что его нельзя вложить ни в какое инвариантное конечномерное липшицево подмногообразие (см. [32] по поводу деталей). Таким образом, основным результатом этого раздела является следующая теорема.

ТеОрема 3.1. Пусть $A$ - самосопряженный положительный оператор в $H$ с компактным обратным, и пусть константа $L_{0}$, определяющая максимальную величину щели в спектре (см. (2.2)), конечна $\left(L_{0}<\infty\right)$. Тогда для любого $L>\max \left\{L_{0} / 2, \lambda_{2}\right\}$ существует гладкая нелинейность $F(u)$, удовлетворяющая (2.3), такая, что соответствующая задача (2.1) имеет глобальный аттрактор $\mathscr{A}$, который содержит две различные траектории и $(t)$ u v(t), удовлетворяющие (3.1). 
ДокАЗАтЕльство. Конструкция нелинейности $F(u)$ с описанными выше свойствами базируется на некотором обобщении известного контрпримера к теории Флоке в бесконечномерных пространствах (см. [5], [15]). Для этого сначала будет построен периодический по времени оператор $\Phi(t)$ такой, что его норма сколь угодно близка к константе щели в спектре $L_{0}$ и все решения уравнения

$$
\partial_{t} w+A w=\Phi(t) w
$$

убывают быстрее чем экспоненциально, когда время стремится к бесконечности.

Для построения этого оператора нам понадобится скалярная периодическая функция $x(t)$ с периодом $2 T$ ( $T$ - произвольное достаточно большое число), удовлетворяющая следующим условиям:

1) $x(-t)=-x(t)$ и $x(T-t)=x(t)$ для любого $t$,

2) $x(T / 2):=N \geqslant 1$ является ее максимальным значением,

3) $x^{\prime \prime}(t)<0$ при $0<t<T$ и $x^{\prime}(t)>0$ при $0<t<T / 2$.

Такой функцией является, например, $x(t)=\sin (\pi t / T)$.

Без ограничения общности можно предположить также, что собственные значения оператора $A$ удовлетворяют условию

$$
c_{2} k \leqslant \lambda_{k} \leqslant c_{1} k
$$

для некоторых положительных $c_{1}$ и $c_{2}$. Действительно, отсутствие щели в спектре $\left(L_{0}<\infty\right)$ дает оценку сверху для $\lambda_{k}$, а оценку снизу можно получить, просто выбрасывая из рассмотрения слишком плотно расположенные собственные значения (проекция нелинейности на эти моды будет равна нулю тождественно).

Следующее предложение играет ключевую роль в дальнейшем изложении.

ПредлОЖенИЕ 3.2. Пусть выполнены условия теоремы 2.1. Тогда для любого $L>L_{0} / 2$ и любой периодической функиии $x(t)$, удовлетворяющей сформулированным выше условиям, существует гладкое отображсние $\mathscr{R}: \mathbb{R} \rightarrow$ $\mathscr{L}(H, H)$ maкое, что

$$
\|\mathscr{R}(x)\|_{\mathscr{L}(H, H)} \leqslant L .
$$

Более того, если определить $2 T$-периодическое отображение формулой $\Phi(t):=$ $\mathscr{R}(x(t))$, то отображение за период $P:=U(2 T, 0)$, где $U(t, s)$ - разрешаюиий оператор уравнения $(3.2)(u(t)=U(t, s) u(s))$, удовлетворяет следующим условиям:

$$
\begin{gathered}
P e_{2 n-1}=\mu_{2 n-1} e_{2 n+1}, \quad n \in \mathbb{N}, \quad P e_{2 n}=\mu_{2 n-2} e_{2 n-2}, \quad n>1, \\
P e_{2}=\mu_{0} e_{1},
\end{gathered}
$$

где положительные мультипликаторы $\mu_{n}, \mu_{0}$ определяются формулами

$$
\mu_{n}:=e^{-T\left(\lambda_{n}+2 \lambda_{n+1}+\lambda_{n+2}\right) / 2}, \quad \mu_{0}:=e^{-T\left(2 \lambda_{1}+\lambda_{2}\right) / 2} .
$$

В частности, все решения уравнения (3.2) убъвают быстрее чем экспоненииально:

$$
\|w(t)\|_{H} \leqslant C e^{-\beta t^{2}}\|w(0)\|_{H},
$$

и положительные константы $C$ и $\beta$ не зависят от $w(0) \in H$. 
ДокАзАтЕльство. Введем пару гладких срезающих функций $\theta_{1}(x)$ и $\theta_{2}(x)$ таких, что

1) $\theta_{1}(x) \equiv 1$ при $x \in[N / 2, N]$ и $\theta_{1}(x) \equiv 0$ при $x \leqslant N / 4$;

2) $\theta_{2}(x) \equiv 1$ при $x \geqslant N / 4$ и $\theta_{2}(x) \equiv 0$ при $x \leqslant 0$.

Определим также линейные операторы $F^{ \pm}$формулами

$$
\begin{aligned}
F_{2 n-1}^{-}(x) u & =\frac{1}{2}\left(\lambda_{2 n-1}-\lambda_{2 n}\right) u_{2 n-1} \theta_{2}(x)+\varepsilon \theta_{1}(x) u_{2 n}, \\
F_{2 n}^{-}(x) u & =-\frac{1}{2}\left(\lambda_{2 n-1}-\lambda_{2 n}\right) u_{2 n} \theta_{2}(x)-\varepsilon u_{2 n-1} \theta_{1}(x), \quad n \in \mathbb{N},
\end{aligned}
$$

И

$$
\begin{aligned}
F_{1}^{+}(x) u & =0 \\
F_{2 n}^{+}(x) u & =\frac{1}{2}\left(\lambda_{2 n}-\lambda_{2 n+1}\right) u_{2 n} \theta_{2}(-x)+\varepsilon u_{2 n+1} \theta_{1}(-x), \\
F_{2 n+1}^{+}(x) u & =-\frac{1}{2}\left(\lambda_{2 n}-\lambda_{2 n+1}\right) u_{2 n+1} \theta_{2}(-x)-\varepsilon u_{2 n} \theta_{1}(-x), \quad n \in \mathbb{N},
\end{aligned}
$$

где $\varepsilon>0$ - положительное число, которое будет определено позднее (см. формулу (3.16) ниже). Наконец, определим искомый оператор $\Phi(t)$ следующим образом:

$$
\Phi(x(t)) u:=F^{+}(x(t)) u+F^{-}(x(t)) u .
$$

Мы утверждаем, что существует малое $\varepsilon=\varepsilon(T)>0$ такое, что определенный выше оператор удовлетворяет всем условиям предложения. Действительно, пусть $P_{-}$и $P_{+}-$разрешающие операторы, которые отображают $w(0)$ в $w(T)$ и $w(T)$ в $w(2 T)$ соответственно:

$$
P_{-}:=U(T, 0), \quad P_{+}:=U(2 T, T) .
$$

Тогда двумерные подпространства

$$
V_{n}^{-}:=\operatorname{span}\left\{e_{2 n-1}, e_{2 n}\right\}, \quad V_{n}^{+}:=\operatorname{span}\left\{e_{2 n}, e_{2 n+1}\right\}, \quad n \in \mathbb{N},
$$

инвариантны относительно $P_{-}$и $P_{+}$соответственно.

Необходимо найти образы $P_{ \pm} e_{n}$ координатных векторов под действием этих отображений. Для этого введем минимальное положительное $T_{0}$ такое, что $x\left(T_{0}\right)=N / 4$. Тогда, согласно определению срезающих функций и оператора $\Phi$, все координатные векторы $e_{n}$ инвариантны относительно разрешающих операторов $U\left(T_{0}, 0\right), U\left(T, T-T_{0}\right), U\left(T+T_{0}, T\right)$ и $U\left(2 T, 2 T-T_{0}\right)$. Таким образом, необходимо изучить только операторы $U\left(T-T_{0}, T_{0}\right)$ и $U\left(2 T-T_{0}, T+T_{0}\right)$. На этих интервалах времени срезающая функция $\theta_{2}$ тождественно равна 1 , что ведет к следующим уравнениям:

$$
\begin{aligned}
\frac{d}{d t} u_{2 n-1} & =-\frac{1}{2}\left(\lambda_{2 n-1}+\lambda_{2 n}\right) u_{2 n-1}+\varepsilon \theta_{1}(x(t)) u_{2 n}, \\
\frac{d}{d t} u_{2 n} & =-\frac{1}{2}\left(\lambda_{2 n-1}+\lambda_{2 n}\right) u_{2 n}-\varepsilon \theta_{1}(x(t)) u_{2 n-1}
\end{aligned}
$$


при $t \in\left[T_{0}, T-T_{0}\right]$ и

$$
\begin{aligned}
\frac{d}{d t} u_{2 n} & =-\frac{1}{2}\left(\lambda_{2 n}+\lambda_{2 n+1}\right) u_{2 n}+\varepsilon \theta_{1}(-x(t)) u_{2 n+1}, \\
\frac{d}{d t} u_{2 n+1} & =-\frac{1}{2}\left(\lambda_{2 n}+\lambda_{2 n+1}\right) u_{2 n+1}-\varepsilon \theta_{1}(-x(t)) u_{2 n}
\end{aligned}
$$

при $t \in\left[T+T_{0}, 2 T-T_{0}\right]$. Для изучения решений этих уравнений удобно ввести полярные координаты

$$
u_{2 n-1}+i u_{2 n}=R_{n}^{-} e^{i \varphi_{n}^{-}}, \quad u_{2 n}+i u_{2 n+1}=R_{n}^{+} e^{i \varphi_{n}^{+}}
$$

(для уравнений (3.12) и (3.13) соответственно). Тогда для фаз $\varphi_{n}^{ \pm}$получаем

$$
\frac{d}{d t} \varphi_{n}^{-}=-\varepsilon \theta_{1}(x(t)) \quad \text { и } \quad \frac{d}{d t} \varphi_{n}^{+}=-\varepsilon \theta_{1}(-x(t))
$$

при $t \in\left[T_{0}, T-T_{0}\right]$ и $t \in\left[T+T_{0}, 2 T-T_{0}\right]$ соответственно.

Наконец, зафиксируем

$$
\varepsilon:=-\frac{\pi}{2 \int_{T_{0}}^{T-T_{0}} \theta_{1}(x(t)) d t}=-\frac{\pi}{2 \int_{T+T_{0}}^{2 T-T_{0}} \theta_{1}(-x(t)) d t} .
$$

Тогда оба оператора $U\left(T-T_{0}, T_{0}\right)$ и $U\left(2 T-T_{0}, T+T_{0}\right)$, суженные на $V_{n}^{-}$и $V_{n}^{+}$соответственно, будут представляться в виде композиции поворота на $\pi / 2$ и сжатия. А именно,

$$
\begin{aligned}
U\left(T-T_{0}, T_{0}\right) e_{2 n-1} & =e^{-\left(\left(T-2 T_{0}\right) / 2\right)\left(\lambda_{2 n-1}+\lambda_{2 n}\right)} e_{2 n}, \\
U\left(T-T_{0}, T_{0}\right) e_{2 n} & =e^{-\left(\left(T-2 T_{0}\right) / 2\right)\left(\lambda_{2 n-1}+\lambda_{2 n}\right)} e_{2 n-1}, \\
U\left(2 T-T_{0}, T+T_{0}\right) e_{2 n} & =e^{-\left(\left(T-2 T_{0}\right) / 2\right)\left(\lambda_{2 n}+\lambda_{2 n+1}\right)} e_{2 n+1}, \\
U\left(2 T-T_{0}, T+T_{0}\right) e_{2 n+1} & =e^{-\left(\left(T-2 T_{0}\right) / 2\right)\left(\lambda_{2 n}+\lambda_{2 n+1}\right)} e_{2 n} .
\end{aligned}
$$

Более того, при $t \in\left[0, T_{0}\right]$ и $t \in\left[T-T_{0}, T_{0}\right]$ уравнения распадаются:

$$
\begin{aligned}
\frac{d}{d t} u_{2 n-1}+\lambda_{2 n-1} u_{2 n-1} & =\frac{1}{2}\left(\lambda_{2 n-1}-\lambda_{2 n}\right) \theta_{2}(x(t)) u_{2 n-1}, \\
\frac{d}{d t} u_{2 n}+\lambda_{2 n} u_{2 n} & =-\frac{1}{2}\left(\lambda_{2 n-1}-\lambda_{2 n}\right) \theta_{2}(x(t)) u_{2 n},
\end{aligned}
$$

и, следовательно,

$$
\begin{aligned}
U\left(T_{0}, 0\right) e_{2 n-1} & =e^{-\lambda_{2 n-1} T_{0}} e^{(1 / 2)\left(\lambda_{2 n-1}-\lambda_{2 n}\right) \int_{0}^{T_{0}} \theta_{2}(x(t)) d t} e_{2 n-1}, \\
U\left(T_{0}, 0\right) e_{2 n} & =e^{-\lambda_{2 n} T_{0}} e^{-(1 / 2)\left(\lambda_{2 n-1}-\lambda_{2 n}\right) \int_{0}^{T_{0}} \theta_{2}(x(t)) d t} e_{2 n}, \\
U\left(T, T-T_{0}\right) e_{2 n-1} & =e^{-\lambda_{2 n-1} T_{0}} e^{(1 / 2)\left(\lambda_{2 n-1}-\lambda_{2 n}\right) \int_{0}^{T_{0}} \theta_{2}(x(t)) d t} e_{2 n-1}, \\
U\left(T, T-T_{0}\right) e_{2 n} & =e^{-\lambda_{2 n} T_{0}} e^{-(1 / 2)\left(\lambda_{2 n-1}-\lambda_{2 n}\right) \int_{0}^{T_{0}} \theta_{2}(x(t)) d t} e_{2 n} .
\end{aligned}
$$


Таким образом, для оператора $P_{-}=U\left(T, T-T_{0}\right) U\left(T-T_{0}, T_{0}\right) U\left(T_{0}, 0\right)$ получаем, что

$$
\begin{aligned}
P_{-} e_{2 n-1}= & e^{-\lambda_{2 n-1} T_{0}} e^{(1 / 2)\left(\lambda_{2 n-1}-\lambda_{2 n}\right) \int_{0}^{T_{0}} \theta_{2}(x(t)) d t} e^{-\left(\left(T-2 T_{0}\right) / 2\right)\left(\lambda_{2 n-1}+\lambda_{2 n}\right)} \\
& \quad \times e^{-\lambda_{2 n} T_{0}} e^{-(1 / 2)\left(\lambda_{2 n-1}-\lambda_{2 n}\right) \int_{0}^{T_{0}} \theta_{2}(x(t)) d t} e_{2 n} \\
= & e^{-T\left(\lambda_{2 n-1}+\lambda_{2 n}\right) / 2} e_{2 n}, \\
P_{-} e_{2 n}= & e^{-\lambda_{2 n} T_{0}} e^{-(1 / 2)\left(\lambda_{2 n-1}-\lambda_{2 n}\right) \int_{0}^{T_{0}} \theta_{2}(x(t)) d t} e^{-\left(\left(T-2 T_{0}\right) / 2\right)\left(\lambda_{2 n-1}+\lambda_{2 n}\right)} \\
& \quad \times e^{-\lambda_{2 n-1} T_{0}} e^{(1 / 2)\left(\lambda_{2 n-1}-\lambda_{2 n}\right) \int_{0}^{T_{0}} \theta_{2}(x(t)) d t} e_{2 n-1} \\
= & e^{-T\left(\lambda_{2 n-1}+\lambda_{2 n}\right) / 2} e_{2 n-1} .
\end{aligned}
$$

Аналогичные рассуждения для оператора $P_{+}=U\left(2 T, 2 T-T_{0}\right) U\left(2 T-T_{0}, T+\right.$ $\left.T_{0}\right) U\left(T+T_{0}, T\right)$ приводят к формулам

$$
\begin{gathered}
P_{+} e_{1}=e^{-T \lambda_{1} / 2} e_{1}, \quad P_{+} e_{2 n}=e^{-T\left(\lambda_{2 n}+\lambda_{2 n+1}\right) / 2} e_{2 n+1}, \\
P_{+} e_{2 n+1}=e^{-T\left(\lambda_{2 n}+\lambda_{2 n+1}\right) / 2} e_{2 n},
\end{gathered}
$$

$n \in \mathbb{N}$. Это доказывает сформулированные в предложении спектральные свойства оператора $P:=P_{+} \circ P_{-}$(см. (3.5)).

Заметим также, что, благодаря (3.16), $\varepsilon \rightarrow 0$ при $T \rightarrow \infty$, а норма оператора $\Phi(x(t))$ при $\varepsilon=0$, очевидно, не превосходит $L_{0} / 2$. Более того, константа $\varepsilon$, определенная формулой (3.16), может быть сделана сколь угодно малой за счет увеличения периода $T$. Действительно, в силу предположения о выпуклости функции $x(t)$ имеет место неравенство $x(t) \geqslant N / 2$ при $t \in[T / 4,3 T / 2]$. Поэтому

$$
|\varepsilon| \leqslant \frac{\pi}{T}
$$

Таким образом, (3.4) будет выполнено при достаточно большом $T$.

Остается проверить (3.7). Для этого достаточно доказать, что

$$
\left\|P^{N} e_{2 n}\right\|_{H} \leqslant C e^{-\beta_{1} N^{2}}
$$

равномерно по всем $n \in \mathbb{N}$. Действительно, согласно (3.6) и (3.3), мультипликаторы $\mu_{n}$ удовлетворяют неравенствам

$$
e^{-C_{2} T n} \leqslant \mu_{n} \leqslant e^{-C_{1} T n}
$$

Поэтому при $N \geqslant n$

$$
\left\|P^{N} e_{2 n}\right\| \leqslant e^{-C T\left(\sum_{k=0}^{n} 2 k+\sum_{k=0}^{N-n} 2 k\right)}=e^{-C T(n(n+1)+(N-n)(N-n+1))} \leqslant e^{-C T N^{2} / 2} .
$$

Так как при $N \leqslant n$ неравенство (3.19) очевидно, проверка (3.7) закончена, и предложение 3.2 доказано.

Теперь мы можем завершить построение искомого контрпримера. Для этого рассмотрим $2 T$-периодическую траекторию $(x(t), y(t))$, порожденную двумерной системой ОДУ:

$$
\frac{d}{d t} x=f(x, y), \quad \frac{d}{d t} y=g(x, y)
$$


с гладкими правыми частями $f$ и $g$ (которые можно обрезать при больших $x$ и $y$ так, чтобы получить диссипативность), и предположим, что ее первая компонента $x(t)$ удовлетворяет перечисленным выше условиям. Перенормируя время (и увеличивая период $T$ ), мы можем также сделать константы Липшица для функций $f, g$ и $x$ произвольно малыми.

Рассмотрим следующую систему уравнений для $u=(x, y, w)$ :

$$
\frac{d}{d t} x=f(x, y), \quad \frac{d}{d t} y=g(x, y), \quad \partial_{t} w+A w=\mathscr{R}(x) w .
$$

Очевидно, что система (3.22) имеет вид (2.1) (нужно только зарезервировать первые две моды $e_{1}$ и $e_{2}$ для функций $x$ и $y$ и переобозначить оператор $Q_{3} A$ через $A$ ). Также нетрудно видеть, что липшицева норма нелинейности в системе (3.22) может быть сделана сколь угодно близкой к $L>L_{0} / 2$ (так как константы Липшица функций $f, g$ и $x$ малы), но для того чтобы построить периодическую траекторию $x(t), y(t)$, нужно иметь возможность компенсировать диссипативные члены $\lambda_{1} x$ и $\lambda_{2} y$, приходящие из линейной части уравнения (2.1), а это можно сделать благодаря дополнительному условию $L>\lambda_{2}$.

Для завершения построения нам нужно гарантировать, что хотя бы одна из полутраекторий вида $v(t):=(x(t), y(t), w(t)), t \geqslant 0$, с ненулевым $w(t)$ лежит на аттракторе. Для этого зафиксируем решение $v(t)$ системы (3.22) такое, что $w(0)=e_{1}$ и $w_{1}(t):=\left(w(t), e_{1}\right)<1$ для всех $t \geqslant 0$. После этого зададим малое гладкое возмущение $R\left(x, y, w_{1}\right)=\left(R_{1}, R_{2}, R_{3}\right)$ и рассмотрим возмущенный аналог системы (3.22)

$$
\begin{gathered}
\frac{d}{d t} x=f(x, y)+R_{1}\left(x, y, w_{1}\right), \quad \frac{d}{d t} y=g(x, y)+R_{2}\left(x, y, w_{1}\right), \\
\frac{d}{d t} w+A w=\mathscr{R}(x) w+R_{3}\left(x, y, w_{1}\right) e_{1}
\end{gathered}
$$

такой, что $R \equiv 0$ при $w_{1} \leqslant 2$ и, кроме того, трехмерная система

$$
\begin{gathered}
\frac{d}{d t} x=f(x, y)+R_{1}\left(x, y, w_{1}\right), \quad \frac{d}{d t} y=g(x, y)+R_{2}\left(x, y, w_{1}\right), \\
\frac{d}{d t} w_{1}+\lambda_{1} w_{1}=R_{3}\left(x, y, w_{1}\right)
\end{gathered}
$$

имеет седловую точку (где-то в области $w_{1}>2$ и $x<0$ ) такую, что точка $(x(0), y(0), 1)$ принадлежит неустойчивому многообразию этой седловой точки, а соответствующая ей траектория $v(t)=\left(\bar{x}(t), \bar{y}(t), w_{1}(t)\right)$, выходящая из седла при $t \rightarrow-\infty$, удовлетворяет условию $\bar{x}(t)<0$ при $t<0$. Такое возмущение, очевидно, существует. Более того, используя трюк с перенормировкой времени, можно добиться, чтобы константа Липшица нелинейности в (3.23) не превосходила $L$.

Далее, предположение $\bar{x}(t)<0$ при $t<0$ гарантирует, что $F^{-}(\bar{x}(t)) \equiv 0$ при $t \leqslant 0$ (см. определение этого оператора в (3.8)), и, следовательно, прямая $\mathbb{R} e_{1}$ является инвариантной для оператора $\mathscr{R}(\bar{x}(t))$ при $t<0$. По этой причине решение $\left(\bar{x}(t), \bar{y}(t), w_{1}(t)\right), t \leqslant 0$, порождает решение $(\bar{x}(t), \bar{y}(t), w(t)), t \leqslant 0$, для бесконечномерной системы (3.24) (следует просто положить $w_{i}(t)=0$ при 
$i>1)$. А так как $x(0)=\bar{x}(0)$ и $y(0)=\bar{y}(0)$ и нелинейность $R$ тождественно равна нулю на этой траектории при $t \geqslant 0$, то $\bar{x}(t)=x(t)$ и $\bar{y}(t)=y(t)$ при $t \geqslant 0$.

Наконец, так как любое неустойчивое многообразие и любая периодическая орбита всегда принадлежат глобальному аттрактору, обе траектории $v(t)$ и $u(t):=(x(t), y(t), 0)$ лежат на аттракторе. Остается заметить, что, согласно предложению 3.2 ,

$$
\|u(t)-v(t)\|=\|w(t)\| \leqslant C e^{-\beta t^{2}} .
$$

Теорема 3.1 доказана.

\section{4. Отсутствие лог-липшицевых вложений}

В этом разделе будет показано, что при выполнении условий теоремы 3.1 можно построить гладкую нелинейность $F(u)$ таким образом, чтобы соответствующий аттрактор $\mathscr{A}$ не вкладывался ни в какое конечномерное лог-липшицево многообразие.

Напомним, что отображение $T: X \rightarrow Y$ между двумя метрическими пространствами $X$ и $Y$ называется лог-липшицевым (или $\gamma$-лог-липшицевым), если существуют константы $\gamma \in(0, \infty)$ и $C>0$ такие, что

$$
d\left(T x_{1}, T x_{2}\right) \leqslant C d\left(x_{1}, x_{2}\right)\left(\log \frac{C}{d\left(x_{1}, x_{2}\right)}\right)^{\gamma}
$$

для всех $x_{1}, x_{2} \in X$. Отображение $T$ называется лог-липшицевым гомеоморфизмом, если оба отображения $T$ и $T^{-1}$ являются лог-липшицевыми, и топологическое пространство $\mathscr{M}$ называется лог-липшицевым многообразием над $\mathbb{R}^{N}$, если оно локально гомеоморфно $\mathbb{R}^{N}$ и все координатные отображения лог-липшицевы.

Для построения искомого контрпримера нам понадобится еще несколько определений и вспомогательных утверждений.

ОПРЕДЕЛЕНИЕ 4.1. Пусть $X$ - компактное метрическое пространство. Тогда любое его подмножество $B \subset X$ предкомпактно и, согласно критерию Хаусдорфа, может быть покрыто конечным числом $\varepsilon$-шаров для любого $\varepsilon>0$. Обозначим через $N_{\varepsilon}(B, X)$ минимальное число $\varepsilon$-шаров, покрывающих $X$. Тогда, как известно, фрактальная размерность множества $B$ определяется формулой

$$
\operatorname{dim}_{\mathrm{f}}(B, X)=\limsup _{\varepsilon \rightarrow 0} \frac{\log N_{\varepsilon}(B, X)}{\log (1 / \varepsilon)} .
$$

Определим также (эпсилон-) фактор удвоения $D_{\varepsilon}(X)$ следующим образом:

$$
D_{\varepsilon}(X):=\sup _{x \in X} N_{\varepsilon / 2}(B(\varepsilon, x)),
$$

где символом $B(r, x)$ обозначен шар радиуса $r$ в $X$ с центром в $x \in X$.

Нетрудно видеть, что $D_{\varepsilon}(X) \leqslant D_{N}<\infty$ при $\varepsilon \rightarrow 0$, если $X$ является подмножеством $\mathbb{R}^{N}$, и, следовательно, условие

$$
D(X):=\sup _{\varepsilon>0} D_{\varepsilon}(X)<\infty
$$


является необходимым (но не достаточным) для существования липшицева вложения $X$ в конечномерное липшищево многообразие (см., например, [24], [29]). Следующая лемма дает аналогичное необходимое условие для лог-липшицева случая.

Лемма 4.2. Пусть $X$ - компактное метрическое пространство, вложенное (при помощи лог-липшицева гомеоморфизма) в лог-липшицево многообразие $M$. Тогда величина

$$
\operatorname{dim}_{\text {Log-D }}(X):=\limsup _{\varepsilon \rightarrow 0} \frac{\log D_{\varepsilon}(X)}{\log \log (1 / \varepsilon)},
$$

называемая далее логарифмическим фактором удвоения, является конечной:

$$
\operatorname{dim}_{\log -\mathrm{D}}(X)<\infty .
$$

ДокАЗАтельство. Очевидно, что нужно оценивать величину (4.5) только для малых $\varepsilon$, т. е. нам нужно оценить число $\varepsilon / 2$-шаров, покрывающих некоторый шар $B(\varepsilon, x)$ с центром в $x \in X$ при $\varepsilon \ll 1$. Благодаря компактности, мы можем предположить без ограничения общности, что $B(\varepsilon, x)$ и все покрывающие его $\varepsilon / 2$-шары лежат в одной и той же координатной карте. Тогда существует биективное отображение $T$ некоторой окрестности $V \supset B(\varepsilon, x)$ в открытое подмножество $\mathbb{R}^{N}$ такое, что $T$ и $T^{-1}$ удовлетворяют (4.1). Таким образом, согласно (4.1),

$$
T^{-1} B(\varepsilon, x) \subset B\left(\varepsilon_{1}, T^{-1} x\right), \quad T B\left(\varepsilon_{2}, y\right) \subset B\left(\frac{\varepsilon}{2}, T y\right), \quad y \in T^{-1} V,
$$

где

$$
\varepsilon_{1}:=C \varepsilon\left(\log \frac{C}{\varepsilon}\right)^{\gamma}, \quad C \varepsilon_{2}\left(\log \frac{C}{\varepsilon_{2}}\right)^{\gamma}=\frac{\varepsilon}{2} .
$$

Поэтому любое покрытие шара $B\left(\varepsilon_{1}, T^{-1} x\right)$ шарами радиуса $\varepsilon_{2}$ в $\mathbb{R}^{N}$ порождает $\varepsilon / 2$-покрытие шара $B(\varepsilon, x)$ в $X$ и, следовательно,

$$
N_{\varepsilon / 2}(B(\varepsilon, x)) \leqslant N_{\varepsilon_{2}}\left(B\left(\varepsilon_{1}, T^{-1} x\right)\right) \leqslant C_{1}\left(\frac{\varepsilon_{1}}{\varepsilon_{2}}\right)^{N},
$$

где константа $C_{1}$ не зависит от $\varepsilon_{i}$ и $T^{-1} x$, благодаря инвариантности $\mathbb{R}^{N}$ по отношению к сдвигам и растяжениям. Из (4.8) и (4.7) можно заключить, что

$$
N_{\varepsilon / 2}(B(\varepsilon, x)) \leqslant C_{2}\left(\log \frac{C}{\varepsilon}\right)^{2 \gamma N} .
$$

Это неравенство доказывает (4.5) и завершает доказательство леммы.

Теперь мы готовы сформулировать основной результат этого раздела.

ТеОрема 4.3. Пусть выполнены условия теоремы 3.1. Тогда для любого показателя $L>\max \left\{L_{0} / 2, \lambda_{2}\right\}$ существует гладкая $\left(C^{\infty}\right.$-гладкая) нелинейность $F(u)$ с глобальной константой Липшица $L$ такая, что глобальный аттрактор $\mathscr{A}$ соответствующей задачи (2.1) имеет бесконечный логарифмический фактор удвоения:

$$
\operatorname{dim}_{\text {Log-D }}(\mathscr{A})=\infty .
$$


$B$ частности, аттрактор $\mathscr{A}$ не допускает конечномерных лог-липшицевых проекиии Мане и не может быть вложен ни в какое конечномерное лог-липшицево многообразие.

ДокАЗАТЕЛЬСтво. Мы будем использовать конструкцию контрпримера, приведенную в теореме 3.1. Но теперь нам будет нужно, чтобы не только одна точка $v(0)=\left(x(0), y(0), e_{1}\right)$, но и целый одномерный отрезок $v_{s}(0):=$ $\left(x(0), y(0), s e_{1}\right), s \in[1,1-\kappa]$, для некоторого $\kappa<1$, лежал в аттракторе. Тогда у нас будет целое семейство траекторий $v_{s}(t)$ на аттракторе, притягивающихся с суперэкспоненциальной скоростью к периодической траектории $u(t)=(x(t), y(t), 0)$. Для достижения этой цели достаточно подправить трехмерную систему ОДУ (3.24) таким образом, чтобы двумерное неустойчивое многообразие $\mathscr{M}_{+}$седловой точки содержало не только $(x(0), y(0), 1)$, но и целый отрезок $v_{s}(0), s \in[1-\kappa, 1]$. Нетрудно показать, что такое $R$ существует, и даже предъявить явную конструкцию - мы оставляем это читателю и предполагаем с данного момента, что система (3.23) выбрана так, чтобы $v_{s}(t)$ принадлежали аттрактору $\mathscr{A}$ для любого $s \in[1-\kappa, 1]$.

Как и большинство контрпримеров в теории размерности и проекций Мане (см. [24], [29] и цитируемую там литературу), наш контрпример также использует (почти) ортогональные последовательности с "плохими" свойствами. Однако, в отличие от упомянутых выше работ, нам необходимо еще и вложить подобные последовательности в аттрактор.

Грубо говоря, мы сформируем прототип такой последовательности, правильно подбирая малые возмущения траекторий $v_{s}(t)$ вблизи $t=0$ в ортогональных направлениях $e_{2 n}$, зависящих от $s$. Конечно, это все еще далеко от ортогональной последовательности, так как все ее элементы имеют большую первую компоненту $w_{1}$. Далее, мы существенно используем тот факт, что первая компонента $w_{1}(t)$ решения убывает "намного быстрее" остальных, поэтому через некоторое правильно выбранное время эта компонента станет пренебрежимо малой по отношению к остальным, и почти ортогональная последовательность с плохими свойствами будет сформирована.

Для того чтобы сделать описанную выше конструкцию строгой, нам понадобится следующая лемма.

Лемма 4.4. Пусть $P$ - отображение за период, порожденное задачей (3.2). Тогда для любого $n \in \mathbb{N} u$ любых $n \leqslant s_{1}, s_{2} \leqslant n+k$, где $k:=[\sqrt{n}] u N=2 n+k$, справедливы следующие оченки:

$$
\frac{\left\|P^{N} e_{1}\right\|}{\left\|P^{N} e_{2 s_{1}}\right\|} \leqslant e^{-\beta n^{2}}, \quad e^{-\gamma n^{3 / 2}} \leqslant \frac{\left\|P^{N} e_{2 s_{1}}\right\|}{\left\|P^{N} e_{2 s_{2}}\right\|} \leqslant e^{\gamma n^{3 / 2}}
$$

для некоторых положительных констант $\beta$ и $\gamma$ всех достаточно больuux $n$.

ДоказАТЕЛьство. Действительно, пусть $\delta_{n}:=\left(\lambda_{n}+\lambda_{n+1}\right) T / 2$ и $\delta_{0}:=$ $T \lambda_{1} / 2$. Тогда, согласно (3.5) и (3.6),

$$
\left\|P^{N} e_{1}\right\|=e^{-\sum_{l=1}^{2 N+1} \delta_{l}}, \quad\left\|P^{N} e_{2 s}\right\|=e^{-\sum_{l=0}^{2 s-1} \delta_{l}-\sum_{l=1}^{2(N-s)+1} \delta_{l}}
$$


для всех $n \leqslant s \leqslant n+k$. Так как

$$
\begin{aligned}
\sum_{l=0}^{2 s-1} \delta_{l}+\sum_{l=1}^{2(N-s)+1} \delta_{l} & =\sum_{l=1}^{2 N+1} \delta_{l}-\sum_{l=2(N-s)+2}^{2 N+1} \delta_{l}+\sum_{l=0}^{2 s-1} \delta_{l} \\
& =\sum_{l=1}^{2 N+1} \delta_{l}-\sum_{l=0}^{2 s-1}\left[\delta_{l+2(N-s)+2}-\delta_{l}\right]
\end{aligned}
$$

то, согласно (3.3),

$$
\frac{\left\|P^{N} e_{1}\right\|}{\left\|P^{N} e_{2 s_{1}}\right\|}=e^{-\sum_{l=0}^{2 s-1}\left[\delta_{l+2(N-s)+2}-\delta_{l}\right]} \leqslant e^{-\sum_{l=0}^{2 n}\left[\delta_{2 n+l}-\delta_{l}\right]} \leqslant e^{-\beta n^{2}}
$$

для некоторого положительного $\beta$. Таким образом, первое неравенство (4.10) доказано. Остается проверить второе. Для этого преобразуем левую часть равенства (4.12) следующим образом:

$$
\sum_{l=0}^{2 s-1} \delta_{l}+\sum_{l=1}^{2(N-s)+1} \delta_{l}=\sum_{l=0}^{2 n} \delta_{l}+\sum_{l=1}^{2 n-1} \delta_{l}+\sum_{l=2 n+1}^{2 s-1} \delta_{l}+\sum_{l=2 n}^{2(N-s)+1} \delta_{l}
$$

что доказывает вторую часть неравенств (4.11), так как два первых члена в правой части (4.13) не зависят от $s$, а третий и четвертый члены содержат не более $2 \sqrt{n}$ членов, каждый из которых не превосходит $\delta_{N} \sim C n$. Лемма доказана.

Согласно лемме 4.4, для любых $n \in \mathbb{N}$ и определенных в условиях леммы $k$ и $N(n)$ существуют числа $E_{s}(n), 0 \leqslant s \leqslant k$ такие, что

$$
1 \geqslant E_{s}(n) \geqslant e^{-2 \gamma n^{3 / 2}}
$$

И

$$
\left\|E_{0}(n) P^{N} e_{2 n}\right\|=\left\|E_{1}(n) P^{N} e_{2(n+1)}\right\|=\cdots=\left\|E_{k}(n) P^{N} e_{2(n+k)}\right\|=B(n),
$$

где

$$
e^{-\gamma_{2} n^{2}} \leqslant B(n) \leqslant e^{-\gamma_{1} n^{2}}
$$

для некоторых положительных $\gamma_{i}$.

Теперь мы готовы описать возмущение уравнения (3.23), которое ведет к аттрактору с бесконечным логарифмическим фактором удвоения. Напомним, что трехмерное возмущение $R$ равно нулю тождественно, если $w_{1}<2$. Поэтому без ограничения общности можно считать, что $R\left(v_{s}(t)\right) \equiv 0$ для всех $s \in[1-\kappa, 1]$ и всех $-\kappa \leqslant t \leqslant 0$.

Разобьем отрезок $s \in[1-\kappa, \kappa]$ на счетное число отрезков $I_{n}$ таких, что $\left|I_{n}\right|=$ $\kappa \cdot 2^{-n}\left(\left|I_{n}\right|\right.$ означает длину отрезка $\left.I_{n}\right)$. Далее, разобьем каждый отрезок $I_{n}$ еще на $2^{[\sqrt{n}]}$ равных отрезков $I_{n, p}, p=1, \ldots, 2^{[\sqrt{n}]}$. Тогда, естественно, $\left|I_{n, p}\right| \geqslant \kappa$. $2^{-2 n}$. В каждом интервале $I_{n, p}$ зафиксируем точку $s_{n, p} \in I_{n, p}-$ середину этого интервала и соответствующую траекторию $v_{s, p}(t)$, лежащую на неустойчивом многообразии, так, чтобы

$$
v_{n, p}(0)=\left(x(0), y(0), s_{n, p} e_{1}\right), \quad s_{n, p} \in I_{n, p} .
$$


Далее, введем семейство срезающих функций $\psi_{n, p}(x, y, w)=\psi_{n, p}\left(x, y, w_{1}\right)$ таких, что

$$
\psi_{n, p}\left(v_{n, p}(t)\right) \equiv 1, \quad t \in[-\kappa, 0], \quad \psi_{n, p}\left(v_{n_{1}, p_{1}}(t)\right) \equiv 0, \quad\left(n_{1}, p_{1}\right) \neq(n, p) .
$$

Очевидно, что такие функции существуют. Более того, так как $\left|I_{n, p}\right| \geqslant \kappa \cdot 2^{-2 n}$, эти функции могут быть выбраны так, чтобы выполнялось неравенство

$$
\left\|\psi_{n, p}\right\|_{C^{r}\left(\mathbb{R}^{3}\right)} \leqslant M_{r} \cdot 2^{2 r n} \quad \text { для всех } \quad r \in \mathbb{N},
$$

где константы $M_{r}$ не зависят от $p$ и $n$.

Нам понадобятся еще одна ненулевая срезающая функция $\theta \in C_{0}^{\infty}(\mathbb{R})$ такая, что

$$
\theta(x) \geqslant 0, \quad \theta(x(t)) \equiv 0 \quad \text { для всех } \quad t \in[-T, 0], \quad t \notin(-\kappa, 0),
$$

и семейство констант

$$
K_{n}:=\int_{-\kappa}^{0} e^{-\lambda_{2 n} h} \theta(x(h)) d h .
$$

Очевидно, что

$$
1 \geqslant K_{n} \geqslant 2^{-C n}
$$

для некоторой правильно выбранной константы $C$.

Обозначим также через

$$
g_{p}(n) \in \operatorname{span}\left\{e_{2(n+1)}, \ldots, e_{2(n+[\sqrt{n}])}\right\}, \quad p=1, \ldots, 2^{[\sqrt{n}]},
$$

вершины $[\sqrt{n}]$-мерного единичного гиперкуба $[0,1]^{[\sqrt{n}]}$, занумерованные некоторым образом.

Мы хотим построить искомое гладкое возмущение $T_{n}\left(x, y, w_{1}\right) \in \mathscr{L}(H, H)$ таким образом, чтобы имели место соотношения

$$
\begin{aligned}
v_{n, p}(2(2 n+[\sqrt{n}]) T) & =\left(x(0), y(0), w_{n, p}(2(2 n+[\sqrt{n}]) T)\right) \\
w_{n, p}(2(2 n+[\sqrt{n}]) T) & =\left(w_{n, p}(2(2 n+[\sqrt{n}]) T), e_{1}\right) e_{1}+e^{-\beta n^{2} / 2} B(n) g_{p}(n)
\end{aligned}
$$

для всех $n \in \mathbb{N}$ и всех $p=1, \ldots, 2^{[\sqrt{n}]}$. Это можно сделать по следующей формуле:

$$
\begin{aligned}
T_{n}\left(x, y, w_{1}\right) w=2^{-\beta n^{2} / 2} \sum_{k=1}^{[\sqrt{n}]} \sum_{p=1}^{2^{[\sqrt{n}]}} \theta(x) \psi_{n, p}\left(x, y, w_{1}\right) \\
\times K_{n+k}^{-1} E_{k}(n) g_{p}(n)_{k} w_{2(n+k)} e_{2(n+k)},
\end{aligned}
$$

где через $g_{p}(n)_{k} \in\{0,1\}$ обозначена $e_{2(n+k)}$-я координата вершины $g_{p}(n)$. Действительно, (4.22) следует непосредственно из определения констант $E_{k}(n)$, $B(n), K_{m}$, срезающих функций и того факта, что для нахождения $e_{2(n+k)}$-й координаты образа вершины $g_{p}(n)$ фактически нужно решить линейное уравнение

$$
\frac{d}{d t} w_{2(n+k)}+\lambda_{2(n+k)} w_{2(n+k)}=e^{-\beta n^{2} / 2} K_{2(n+k)}^{-1} E_{k}(n) g_{p}(n)_{k} \theta(x(t))
$$


с $w_{2(n+k)}(-\kappa)=0$ (напомним, что $\left.\psi_{n, p}\left(v_{n, p}(t)\right) \equiv 1\right)$. Более того, благодаря (4.23), (4.21) и (4.18), мы можем сделать вывод, что

$$
\left\|T_{n}\right\|_{C^{r}\left(\mathbb{R}^{3}, \mathscr{L}(H, H)\right)} \leqslant M_{r}^{\prime} e^{-\beta n^{2} / 2} \cdot 2^{k r n},
$$

где положительная константа $k$ не зависит от $n, r \in \mathbb{N}$, а $M_{r}^{\prime}$ зависит только $r$.

Наконец, определим

$$
T\left(x, y, w_{1}\right) w:=\sum_{n=n_{0}}^{\infty} T_{n}\left(x, y, w_{1}\right) w,
$$

где $n_{0}$ - достаточно большое число. Тогда, используя (4.24), мы видим, что возмущение $T$ является $C^{\infty}$-гладким и для любого $r \in \mathbb{N}$ его $C^{r}$-норма может быть сделана сколь угодно малой за счет увеличения $n_{0}$.

Мы утверждаем, что аттрактор $\mathscr{A}$ возмущенного аналога

$$
\begin{gathered}
\frac{d}{d t} x=f(x, y)+R_{1}\left(x, y, w_{1}\right), \quad \frac{d}{d t} y(t)=g(x, y)+R_{2}\left(x, y, w_{1}\right), \\
\frac{d}{d t} w+A w=\mathscr{R}(x) w+R_{3}\left(x, y, w_{1}\right) e_{1}+T\left(x, y, w_{1}\right) w
\end{gathered}
$$

системы (3.25) имеет бесконечный логарифмический фактор удвоения. Доказательство этого факта базируется на формуле (4.22) и неравенстве (4.10). Действительно, согласно (4.22), аттрактор $\mathscr{A}$ содержит последовательность вершин "почти кубов"

$$
\begin{gathered}
u(0)+\left(w_{n, p}(2(2 n+[\sqrt{n}]) T), e_{1}\right) e_{1}+\varepsilon_{n} g_{p}(n) \in \mathscr{A}, \\
n \geqslant n_{0}, \quad p=1, \ldots, 2^{[\sqrt{n}]},
\end{gathered}
$$

где $\varepsilon_{n}:=e^{-\beta n^{2} / 2} B(n)$ и $u(0)=(x(0), y(0), 0)$. Более того, согласно первой оценке (4.10), оценкам (4.14) и (4.21) и определению (4.23),

$$
\left|\left(w_{n, p}(2(2 n+[\sqrt{n}]) T), e_{1}\right)\right| \leqslant e^{-\beta n^{2} / 2} e^{C n^{3 / 2}} \varepsilon_{n} \ll \varepsilon_{n},
$$

если $n_{0}$ достаточно велико. Таким образом, второе слагаемое в (4.27) является несущественным из-за его малости, поэтому, почти не нарушая строгости (но существенно упрощая обозначения), мы будем считать, что

$$
u(0)+\varepsilon_{n} g_{p}(n) \in \mathscr{A}
$$

для всех $n \geqslant n_{0}$ и всех $p=1, \ldots, 2^{[\sqrt{n}]}$. Как показывает следующая лемма, этого достаточно для бесконечности логарифмического фактора удвоения аттрактора $\mathscr{A}$.

Лемма 4.5. Пусть $\mathscr{B} \subset H$ является компактным множеством в гильбертовом пространстве $Н$ таким, что

$$
\varepsilon_{n} g_{p}(n) \in \mathscr{B}
$$

для всех $n \geqslant n_{0}$ и всех $p=1, \ldots, 2^{[\sqrt{n}]}$, где $\varepsilon_{n} \sim e^{-\gamma n^{2}}$ (с некоторым положительным $\gamma$ ). Тогда логарифмический фактор удвоения множества $\mathscr{B}$ бесконечен. 
ДоказАтельство. Действительно, рассмотрим шар $B_{r_{n} \varepsilon_{n}}$ радиуса $r_{n}:=$ $\sqrt{[\sqrt{n}]} / 2$ с центром в центре гиперкуба, порожденного $\varepsilon_{n} g_{p}(n), p=1, \ldots, 2^{[\sqrt{n}]}$. Тогда, очевидно,

$$
\varepsilon_{n} g_{p}(n) \in B_{r_{n} \varepsilon_{n}} \cap \mathscr{B},
$$

но

$$
N_{\varepsilon_{n} / 2}\left(B_{r_{n} \varepsilon_{n}} \cap \mathscr{B}, \mathscr{B}\right) \geqslant 2^{[\sqrt{n}]} .
$$

Используя теперь элементарную оценку

$$
N_{\varepsilon_{n} / 2}\left(B_{r_{n} \varepsilon_{n}} \cap \mathscr{B}, \mathscr{B}\right) \leqslant\left(D_{\varepsilon_{n} / 2}(\mathscr{B})\right)^{\log _{2} r_{n}}
$$

мы видим, что

$$
\log _{2} D_{\varepsilon_{n} / 2}(\mathscr{B}) \geqslant \frac{\sqrt{n}}{\log _{2} r_{n}} \geqslant n^{1 / 4} \sim\left(\frac{1}{\gamma} \log \frac{2}{\varepsilon_{n}}\right)^{1 / 8} .
$$

Таким образом, логарифмический фактор удвоения $\mathscr{B}$ действительно бесконечен, и лемма доказана.

Тот факт, что глобальный аттрактор $\mathscr{A}$ уравнения (4.26) имеет бесконечный логарифмический фактор удвоения, сразу следует из (4.29) и доказанной леммы. Теорема 4.3 доказана.

\section{5. “Неправильные" проекции и случай конечной гладкости}

В этом разделе мы предъявим еще два контрпримера, в некотором смысле связанных с предыдущими. Один из них показывает, что фрактальная размерность аттрактора может зависеть от выбора фазового пространства, если нелинейность $F(u)$ не является $C^{\infty}$-гладкой, а второй демонстрирует, что даже аттрактор, лежащий в конечномерном гладком многообразии, может потерять свойство вложимости в конечномерное лог-липшицево многообразие, если его "неудачно" спроектировать на плоскость конечной коразмерности.

Для построения этих примеров рассмотрим следующую двумерную систему ОДУ:

$$
\left\{\begin{array}{l}
x^{\prime}=-x\left(x^{2}+y^{2}-1\right), \\
y^{\prime}=-y\left(x^{2}+y^{2}-1\right) .
\end{array}\right.
$$

Ее можно переписать в полярных координатах $x+i y=R e^{i \varphi}$ следующим обра3ом:

$$
R^{\prime}=-R\left(R^{2}-1\right), \quad \varphi^{\prime}=0 .
$$

Таким образом, ее глобальный аттрактор $\mathscr{A}_{0} \subset \mathbb{R}^{2}$ состоит из всех точек $R \in$ $[-1,1], \varphi \in[0,2 \pi]$ и $C^{\infty}$-диффеоморфен двумерному конусу.

Разобьем теперь отрезок $\varphi \in[0,2 \pi]$ на бесконечное число меньших отрезков $I_{n}$, где $\left|I_{n}\right|=E_{n}, n=1,2, \ldots$, удовлетворяют

$$
\sum_{n=1}^{\infty} E_{n}<2 \pi
$$


Далее, зафиксируем $\varphi_{n} \in I_{n}$ и семейство срезающих функций $\psi_{n} \in C_{0}^{\infty}(\mathbb{R})$ таких, что $\psi_{n}\left(\varphi_{k}\right)=\delta_{n k}$ и

$$
\left\|\psi_{n}\right\|_{C^{k}(\mathbb{R})} \leqslant C_{k} E_{n}^{-k}, \quad n=1,2, \ldots,
$$

где константы $C_{k}$ не зависят от $n$, а также еще одну срезающую функцию $\theta \in C_{0}^{\infty}(\mathbb{R})$ такую, что

$$
\theta(R) \equiv 0, \quad R \leqslant 0, \quad \theta(R) \equiv 1, \quad R \in\left[\frac{1}{2}, 1\right] .
$$

Введем, наконец, оператор $F: \mathbb{R}^{2} \rightarrow H$ формулой

$$
F(x, y):=\sum_{n=1}^{\infty} B_{n} \theta(x, y) \psi_{n}(x, y) e_{n}
$$

где $B_{n}$ - некоторая монотонно убывающая последовательность, которая будет определена позднее (см. формулировки теорем 5.2 и 5.4), и рассмотрим систему уравнений

$$
\left\{\begin{array}{l}
x^{\prime}=-x\left(x^{2}+y^{2}-1\right), \quad y^{\prime}=-y\left(x^{2}+y^{2}-1\right), \\
\partial_{t} v+A v=F(x, y) .
\end{array}\right.
$$

Некоторые элементарные свойства этой системы сформулированы в следующей лемме.

Лемма 5.1. Пусть, как обычно, $H^{s}:=D\left(A^{s / 2}\right)$ - шкала гильбертовых пространств, порожденная оператором A. Тогда справедливы следующие утверждения.

1) Отображсение $F$, определенное формулой (5.6), принадлежит $C^{k}\left(\mathbb{R}^{2}, H^{s}\right)$, если и только если

$$
\sup _{n \in \mathbb{N}}\left\{B_{n} \lambda_{n}^{s} E_{n}^{-k}\right\}<\infty .
$$

2) Задача (5.7) имеет глобальный аттрактор $\mathscr{A}$ в $H$, который содержит следуюшую последовательность точек:

$$
P_{n}:=\left(\cos \varphi_{n}, \sin \varphi_{n}, B_{n} \lambda_{n}^{-1} e_{n}\right) \in \mathscr{A}
$$

для $n=1,2, \ldots$. Кроме того, обозначим через $Q_{2}: \mathbb{R}^{2} \times H \rightarrow H$ ортопроектор на $v$-компоненту системь (5.7). Тогда проекиия $Q_{2} \mathscr{A}$ содержит следуюшую последовательность отрезков:

$$
S_{n}:=\left\{s e_{n}, s \in\left[0, B_{n} \lambda_{n}^{-1}\right]\right\} \subset Q_{2} \mathscr{A} .
$$

ДокАЗАтельство. Действительно, первое утверждение леммы является немедленным следствием определения (5.6), оценки (5.4) и того факта, что для любых фиксированных $(x, y) \in \mathbb{R}^{2}$ лишь один член в (5.6) может быть ненулевым. Для проверки (5.9) достаточно заметить, что, согласно определению срезающих функций и оператора $F$, точки $P_{n}$ являются положениями равновесия (5.7) для любого $n \in \mathbb{N}$, а (5.10) следует из того, что существует гетероклиническая орбита, соединяющая $\left(\cos \varphi_{n}, \sin \varphi_{n}, 0\right)$ и $P_{n}$, также для любого $n \in \mathbb{N}$. Лемма доказана. 
Заметим, что вложение (5.10) уже показывает, что

$$
\lim _{\varepsilon \rightarrow 0} D_{\varepsilon}\left(Q_{2} \mathscr{A}\right)=\infty
$$

если невозрастающая последовательность $B_{n}$ строго положительна. Поэтому $Q_{2} \mathscr{A}$ не может быть вложено ни в какое конечномерное липшицево многообразие (см. [24]). Следующая теорема показывает, что при более аккуратном выборе последовательности $B_{n}$ вложения в лог-липшицевы многообразия тоже нет.

Tеорема 5.2. Пусть $\lambda_{n} \sim n^{\kappa}, \kappa>0$ (что соответствует случаю, когда $A$ - эллиптический дифференииальный оператор в ограниченной области), и пусть $B_{n} \sim e^{-(\log n)^{2}}, E_{n}:=1 / n^{2}$. Тогда нелинейность $F$ в уравнении $(5.7)$ принадлежит $C^{\infty}\left(\mathbb{R}^{2}, H^{s}\right)$ для любого $s \in \mathbb{R}$, но

$$
\operatorname{dim}_{\log -\mathrm{D}}\left(Q_{2} \mathscr{A}\right)=\infty
$$

$u$, следовательно, $Q_{2} \mathscr{A}$ не вкладывается ни в какое конечномерное лог-липшииево многообразие.

ДокАЗАтельство. Действительно, гладкость $F$ следует из критерия (5.8). Проверим теперь (5.12). Согласно (5.10), для любого $n \in \mathbb{N}$ точки $B_{n} \lambda_{n}^{-1} e_{k}$, $k=1, \ldots, n$, принадлежат $Q_{2} \mathscr{A}$ и, следовательно,

$$
D_{\varepsilon_{n}}\left(Q_{2} \mathscr{A}\right) \geqslant n, \quad \varepsilon_{n}=\lambda_{n}^{-1} B_{n} .
$$

Так как $\varepsilon_{n} \sim n^{-\kappa} e^{-(\log n)^{2}}$, то нетрудно видеть, что $\log n \sim\left(\log \left(1 / \varepsilon_{n}\right)\right)^{2}$, и $(5.12)$ доказано.

ЗАмечание 5.3. Подчеркнем, что (5.12) неверно для самого аттрактора $\mathscr{A}$, а имеет место только для его "неправильной" проекции $Q_{2} \mathscr{A}$. Более того, подправив уравнение (5.7) следующим образом:

$$
\left\{\begin{array}{l}
x^{\prime}=-\beta x\left(x^{2}+y^{2}-1\right), \quad y^{\prime}=-\beta y\left(x^{2}+y^{2}-1\right), \\
\partial_{t} v+A v=\beta F(x, y),
\end{array}\right.
$$

где $\beta \ll 1$ - малое положительное число, получим уравнение, для которого утверждение теоремы 5.2 остается справедливым, но теперь выполнено также условие щели в спектре и, следовательно, существует $C^{1}$-гладкое инерциальное многообразие, диффеоморфное $\mathbb{R}^{2} \ni(x, y)$ и содержащее глобальный аттрактор $\mathscr{A}$ (выбирая $\beta>0$ достаточно малым, можно сделать это многообразие $C^{k}$-гладким для любого $\left.k>0\right)$.

В качестве последнего примера рассмотрим случай, когда оператор $F$ имеет только конечную гладкость. Напомним, прежде всего, что в случае бесконечной гладкости, например когда $F \in C^{\infty}\left(\mathbb{R}^{2}, H^{s}\right)$ для любого $s \in \mathbb{R}_{+}$, согласно свойству сглаживания для параболических уравнений, разрешающий оператор $S(t): \mathbb{R}^{2} \rightarrow H$ уравнения (5.7) обладает следующим свойством:

$$
\left\|S(1) u_{1}-S(1) u_{2}\right\|_{\mathbb{R}^{2} \times H^{s}} \leqslant C_{s}\left\|u_{1}-u_{2}\right\|_{\mathbb{R}^{2} \times H},
$$


$u_{i}=\left(x_{i}, y_{i}, v_{i}\right) \in \mathscr{A}, i=1,2$. Таким образом, фрактальная размерность аттрактора $\mathscr{A}$ одна и та же во всех пространствах $H^{s}$. Однако в случае конечной гладкости $F$ существует критическая экспонента $s_{0}$ (максимальное $s$, для которого выполнено (5.15)) и для больших $s$ нет оснований предполагать, что размерность аттрактора остается неизменной. Следующая теорема показывает, что эта размерность, действительно, может расти и даже стать бесконечной для некоторого $s>s_{0}$.

TEOPEMA 5.4. Пусmь

$$
\lambda_{n} \sim n^{2}, \quad E_{n} \sim \frac{1}{\lambda_{n}^{1 / 2}\left(\log \lambda_{n}\right)^{2}}, \quad B_{n}=\frac{E_{n}}{\log \lambda_{n}} .
$$

Тогда $F \in C\left(\mathbb{R}^{2}, H^{1}\right) \cap C^{1}\left(\mathbb{R}^{2}, H\right)$ и фрактальная размерность $\operatorname{dim}_{\mathrm{f}}\left(\mathscr{A}, \mathbb{R}^{2} \times\right.$ $\left.H^{s}\right)$ аттрактора $\mathscr{A}$ уравнения (5.14) (с $\left.\beta \ll 1\right)$ равна 2 при $s \leqslant 2$ и растет при $s>2$. Более того, аттрактор $\mathscr{A}$ все еще компактен в $\mathbb{R}^{2} \times H^{3}$, но его фрактальная размерность бесконечна:

$$
\operatorname{dim}_{\mathrm{f}}\left(\mathscr{A}, \mathbb{R}^{2} \times H^{3}\right)=\infty
$$

ДокАзАтельство. Действительно, регулярность отображения $F$ следует из критерия (5.8), компактность аттрактора в $H^{3}$ является следствием того факта, что $\lambda_{n}^{3 / 2} \lambda_{n}^{-1} B_{n} \rightarrow 0$ при $n \rightarrow \infty$, и явной конструкции аттрактора. Тот факт, что $\operatorname{dim}_{\mathrm{f}}\left(\mathscr{A}, \mathbb{R}^{2} \times H\right)=2$, следует из существования двумерного инерциального многообразия (условие щели в спектре выполнено, если $\beta$ достаточно мало), а также из существования двумерного неустойчивого многообразия, лежащего на аттракторе. То, что эта размерность остается равной 2 в пространствах $\mathbb{R}^{2} \times H^{s}$ с $s \leqslant 2$, следует из свойства сглаживания (5.15), справедливого для $s \leqslant 2$ (критическая экспонента $s_{0}$ здесь равна 2). Таким образом, остается проверить (5.17).

Так как фрактальная размерность не возрастает при ортогональном проектировании, то, используя (5.10), получим

$$
\begin{aligned}
\operatorname{dim}_{\mathrm{f}}\left(\mathscr{A}, \mathbb{R}^{2} \times H^{3}\right) & \geqslant \operatorname{dim}_{\mathrm{f}}\left(Q_{2} \mathscr{A}, H^{3}\right) \geqslant \operatorname{dim}_{\mathrm{f}}\left(\bigcup_{n=1}^{\infty} S_{n}, H^{3}\right) \\
& \geqslant \operatorname{dim}_{\mathrm{f}}\left(\bigcup_{n=1}^{\infty}\left\{\lambda_{n}^{-1} B_{n} e_{n}\right\}, H^{3}\right) \\
& =\operatorname{dim}_{\mathrm{f}}\left(\bigcup_{n=1}^{\infty}\left\{\lambda_{n}^{-3 / 2} \frac{1}{\left(\log \lambda_{n}\right)^{3}} e_{n}\right\}, H^{3}\right) \\
& =\operatorname{dim}_{\mathrm{f}}\left(\bigcup_{n=1}^{\infty}\left\{\frac{1}{(\log n)^{3}} e_{n}\right\}, H\right)=\infty
\end{aligned}
$$

и теорема доказана.

ЗАмЕчАниЕ 5.5. Используя тот факт, что счетное объединение множеств хаусдорфовой размерности 2 также имеет хаусдорфову размерность 2 , можно 
проверить, что хаусдорфова размерность построенного выше аттрактора равна 2 во всех $H^{s}, s \leqslant 3$ :

$$
\operatorname{dim}_{H}\left(\mathscr{A}, \mathbb{R}^{2} \times H^{3}\right)=2, \quad s \leqslant 3,
$$

и, в частности, при $s=3$ мы имеем случай аттрактора конечной хаусдорфовой и бесконечной фрактальной размерности.

\section{6. Замечания и открытые вопросы}

В этом заключительном разделе мы дадим некоторые комментарии к построенным контрпримерам и сформулируем связанные с ними открытые вопросы.

ЗАмечАниЕ 6.1. Подчеркнем, что большая часть описанных выше контрпримеров построена в предположении, что нелинейная функция $F$ является $C^{\infty}$-гладкой. Было бы интересно построить аналогичные контрпримеры в классах вещественных аналитических функций. В частности, нетрудно видеть, что пример теоремы 2.1 , который показывает отсутствие $C^{1}$-гладкого инерциального многообразия, может быть реализован и в классе аналитических $F$. Однако обобщение примеров, приведенных в разделах 3 и 4 , на аналитические нелинейности не столь очевидно и является интересной задачей.

Еще один естественный вопрос связан с тем, насколько типичной является ситуация, когда нет $C^{1}$-гладкого, липшицева или лог-липшицева инерциального многообразия. Как и в предыдущем случае, ответ более или менее ясен в случае $C^{1}$-гладкости. Действительно, контрпример теоремы 2.1 базируется на грубых спектральных свойствах линеаризации в окрестности двух положений равновесия, которые не могут исчезнуть при малых возмущениях. Таким образом, инерциального многообразия не будет и для любого малого возмущения уравнения (1.4). Как и в предыдущем случае, ответ на этот вопрос не очевиден ни в липшицевом, ни в лог-липшицевом случае.

ЗАмЕчание 6.2. Также необходимо подчеркнуть, что отсутствие гладкости гомеоморфизма $P: \mathscr{A} \rightarrow P \mathscr{A}$ не влечет, вообще говоря, негладкость сопряженной ДС $\widetilde{S}(t)=P S(t) P^{-1}$. Действительно, в современной теории ДС существует множество примеров, когда две гладкие ДС могут быть сопряжены только с помощью негладкого гомеоморфизма (см., например, теоремы типа Гробмана-Хартмана [11], [14]). Таким образом, доказанные результаты о несуществовании липшицевых и лог-липшицевых проекций Мане не позволяют, строго говоря, сделать вывод о "бесконечномерности" или "негладкости" динамики на аттракторе, так как остается возможность того, что гладкая и конечномерная динамика, сопряженная с динамикой на аттракторе, существует, но вложена в бесконечномерное пространство существенно негладким образом. Более того, именно это и происходит во всех примерах данной работы. Действительно, фактическая динамика на построенных аттракторах выглядит тривиальной и маломерной (например, один устойчивый предельный цикл плюс несколько гиперболических положений равновесия с индексами неустойчивости, не превосходящими 2). 
С другой стороны, структура отображения Пуанкаре в окрестности построенного предельного цикла подобна стандартному оператору сдвига в пространстве $l_{2}(\mathbb{Z})$ (см. предложение 3.2 ), что несовместимо, по крайней мере на интуитивном уровне, с конечномерной динамикой. Таким образом, было бы интересно понять, как наличие подобных структур в фазовом пространстве влияет не только на гладкость вложений аттрактора в конечномерные многообразия, но и на динамические характеристики, бифуркации и т.д. Например, важным следующим шагом было бы построение примеров, показывающих, что динамика на аттракторе может быть не сопряжена даже топологически ни с какой гладкой динамикой на компактном множестве $\mathbb{R}^{N}$ (или доказательство того, что такое сопряжение всегда существует). Заметим, что, из-за суперэкспоненциальной сходимости к предельному циклу (см. разделы 3 и 4), такой сопрягающий гомеоморфизм, если и существует, то заведомо не может быть гёльдеровым.

ЗАмечАниЕ 6.3. Построенные контрпримеры, в некотором смысле, демонстрируют естественные предель применимости подхода, связанного с представлением исследуемого УрЧП в виде абстрактной функциональной модели, и показывают, что многие ключевые результаты, полученные к настоящему времени для абстрактного уравнения (1.4), являются точными и не могут быть существенно улучшены. Например, как известно, проекции Мане аттракторов таких уравнений всегда могут быть сделаны гёльдеровыми с показателем Гёльдера, сколь угодно близким к единице, и, как следует из наших примеров, сделать их липшицевыми или лог-липшицевыми, вообще говоря, нельзя. Как мы также увидели, известные препятствия к существованию $C^{1}$-гладких инерциальных многообразий (см. раздел 2) могут препятствовать и существованию липшицевых и лог-липшицевых многообразий, и, таким образом, классическое условие щели в спектре отвечает также за существование менее гладких многообразий (по крайней мере, для случая абстрактных уравнений). Кроме того, из теорем об обратной единственности для параболических уравнений и метода, основанного на логарифмической выпуклости (см. [1], [16]), нетрудно получить следующую $1 / 2$-лог-липшицевость на аттракторе:

$$
\left\|A u_{1}-A u_{2}\right\|_{H} \leqslant C\left\|u_{1}-u_{2}\right\|_{H}\left(\log \frac{C}{\left\|u_{1}-u_{2}\right\|_{H}}\right)^{1 / 2}, \quad u_{1}, u_{2} \in \mathscr{A},
$$

а примеры раздела 3 показывают, что в этой оценке нельзя ожидать лучшего показателя, чем $1 / 2$ (именно экспонента $1 / 2$ дает максимально возможный суперэкспоненциальный рост/убывание расстояния между решениями вида $\left.e^{-t^{2}}\right)$.

Однако из построенных примеров не следует, по крайней мере непосредственно, что идея с липшицевыми или лог-липшицевыми проекциями Мане не будет работать в случае конкретных классов уравнений математической физики, например, для уравнений реакции-диффузии или уравнений Навье-Стокса на торе.

Действительно, чтобы распространить построенные выше контрпримеры с абстрактных уравнений вида (1.4) на конкретные классы УрЧП, например 
на уравнения реакции-диффузии, для начала нужно решить проблему построения положений равновесия $u_{1}$ и $u_{2}$ уравнения

$$
\partial_{t} u=a \Delta_{x} u-f(x, u)
$$

со спектральными свойствами, описанными в разделе 2. Эта проблема, которая является наиболее сложной с нашей точки зрения, тесно связана с нетривиальной и не до конца понятой многомерной обратной спектральной задачей для линейных уравнений вида (6.2). В частности, насколько известно авторам, построение контрпримера к теории Флоке, аналогичного приведенному в разделе 3, для случая линейных параболических УрЧП с периодическими коэффициентами в ограниченных областях является открытой проблемой.

Напомним также, что, в отличие от абстрактных уравнений, условие щели в спектре может и не быть необходимым в случае конкретных классов УрЧП. Так, например, для одномерного уравнения (1.7), упомянутого во введении, условие щели в спектре, вообще говоря, не выполняется (из-за присутствия $u_{x}$ в нелинейности). Однако линеаризованный оператор

$$
L_{u} v:=v_{x} x-f_{u}^{\prime} v-f_{u_{x}}^{\prime} v_{x}
$$

является подобным самосопряженному оператору $w_{x x}-K(x) w$ (где $w=$ $\left.e^{(1 / 2) \int f_{u_{x}}^{\prime} d x} v\right)$, для которого всегда имеется щель в спектре, и, следовательно, контрпример раздела 2 невозможен. Это свойство играет ключевую роль в доказательстве Романова существования липшицевых проекций Мане для аттракторов уравнений этого класса.

Таким образом, представляется важным и интересным найти/изучить другие препятствия, делающие невозможным существование положений равновесия с описанными в разделе 2 спектральными свойствами.

Авторы благодарны Д. В. Тураеву за множество плодотворных дискуссий.

\section{Список литературы}

[1] S. Agmon, L. Nirenberg, "Lower bounds and uniqueness theorems for solutions of differential equations in a Hilbert space", Comm. Pure Appl. Math., 20:1 (1967), 207-229.

[2] А.В. Бабин, М.И. Вишик, Аттракторы эволюиионных уравнений, Наука, М., 1989, 296 с.; англ. изд.: A. V. Babin, M. I. Vishik, Attractors of evolution equations, Stud. Math. Appl., 25, North-Holland Publishing Co., Amsterdam, 1992, x+532 pp.

[3] A. Ben-Artzi, A. Eden, C. Foias, B. Nicolaenko, "Hölder continuity for the inverse of Mañé's projection", J. Math. Anal. Appl., 178:1 (1993), 22-29.

[4] V.V. Chepyzhov, M.I. Vishik, Attractors for equations of mathematical physics, Amer. Math. Soc. Colloq. Publ., 49, Amer. Math. Soc., Providence, RI, 2002, xii+363 pp.

[5] G. Dangelmayr, B. Fiedler, K. Kirchgässner, A. Mielke, Dynamics of nonlinear waves in dissipative systems: reduction, bifurcation and stability, Pitman Res. Notes Math. Ser., 352, Longman, Harlow, 1996, vi+277 pp.

[6] A. Eden, C. Foias, B. Nicolaenko, R. Temam, Exponential attractors for dissipative evolution equations, RAM Res. Appl. Math., 37, Masson, Paris; John Wiley \& Sons, Ltd., Chichester, 1994, viii+183 pp. 
[7] C. Foias, E. Olson, "Finite fractal dimension and Hölder-Lipschitz parametrization", Indiana Univ. Math. J., 45:3 (1996), 603-616.

[8] C. Foias, G. R. Sell, R. Temam, "Inertial manifolds for nonlinear evolutionary equations", J. Differential Equations, 73:2 (1988), 309-353.

[9] C. Foias, G. R. Sell, E.S. Titi, "Exponential tracking and approximation of inertial manifolds for dissipative nonlinear equations", J. Dynam. Differential Equations, 1:2 (1989), 199-244.

[10] А. Ю. Горицкий, В.В.Чепыжов, "Свойство дихотомии решений квазилинейных уравнений в задачах об инерциальных многообразиях", Матем. сб., 196:4 (2005), 23-50; англ. пер.: A. Yu. Goritskii, "Dichotomy property of solutions of quasilinear equations in problems on inertial manifolds", Sb. Math., 196:4 (2005), 485-511.

[11] Ф. Хартман, Обыкновенные дифференциалъные уравнения, Мир, М., 1970, 720 с.; пер. с англ.: Ph. Hartman, Ordinary differential equations, John Wiley \& Sons, Inc., New York-London-Sydney, 1964, xiv+612 pp.

[12] B. R. Hunt, V. Yu. Kaloshin, "Regularity of embeddings of infinite-dimensional fractal sets into finite-dimensional spaces", Nonlinearity, 12:5 (1999), 1263-1275.

[13] L. Kapitanski, I. Rodnianski, "Shape and Morse theory of attractors", Comm. Pure Appl. Math., 53:2 (2000), 218-242.

[14] А. Каток, Б. Хасселблат, Введение в современную теорию динамических систем, Факториал, М., 1999, 767 с.; пер. с англ.: А. Katok, B. Hasselblatt, Introduction to the modern theory of dynamical systems, with a supplementary chapter by A. Katok and L. Mendoza, Encyclopedia Math. Appl., 54, Cambridge Univ. Press, Cambridge, 1995, xviii+802 pp.

[15] P. Kuchment, Floquet theory for partial differential equations, Oper. Theory Adv. Appl., 60, Birkhäuser Verlag, Basel, 1993, xiv+350 pp.

[16] I. Kukavica, "Log-log convexity and backward uniqueness", Proc. Amer. Math. Soc., 135:8 (2007), 2415-2421.

[17] J. Mallet-Paret, G.R. Sell, "Inertial manifolds for reaction diffusion equations in higher space dimensions", J. Amer. Math. Soc., 1:4 (1988), 805-866.

[18] J. Mallet-Paret, G. R. Sell, Zhou De Shao, "Obstructions to the existence of normally hyperbolic inertial manifolds", Indiana Univ. Math. J., 42:3 (1993), 1027-1055.

[19] R. Mañé, "On the dimension of the compact invariant sets of certain nonlinear maps", Dynamical systems and turbulence (Univ. of Warwick, Coventry, 1979/1980), Lecture Notes in Math., 898, Springer, Berlin-New York, 1981, 230-242.

[20] M. Miklavčič, "A sharp condition for existence of an inertial manifold", J. Dynam. Differential Equations, 3:3 (1991), 437-456.

[21] А. Милке, С. В. Зелик, "Бесконечномерные траекторные аттракторы эллиптических краевых задач в цилиндрических областях", УМH, 57:4(346) (2002), 119-150; англ. пер.: A. Mielke, S.V. Zelik, "Infinite-dimensional trajectory attractors of elliptic boundary-value problems in cylindrical domains", Russian Math. Surveys, 57:4 (2002), 753-784.

[22] A. Miranville, S. Zelik, "Attractors for dissipative partial differential equations in bounded and unbounded domains", Handbook of differential equations: evolutionary equations, v. IV, Elsevier/North-Holland, Amsterdam, 2008, 103-200.

[23] H. Movahedi-Lankarani, "On the inverse of Mañé's projection", Proc. Amer. Math. Soc., 116:2 (1992), 555-560.

[24] E. Olson, "Bouligand dimension and almost Lipschitz embeddings", Pacific J. Math., 202:2 (2002), 459-474.

[25] E. J. Olson, J. C. Robinson, "Almost bi-Lipschitz embeddings and almost homogeneous sets", Trans. Amer. Math. Soc., 362:1 (2010), 145-168.

[26] E. Pinto de Moura, J.C. Robinson, "Lipschitz deviation and embeddings of global attractors", Nonlinearity, 23:7 (2010), 1695-1708. 
[27] E. Pinto de Moura, J. C. Robinson, J. J. Sánchez-Gabites, "Embedding of global attractors and their dynamics", Proc. Amer. Math. Soc., 139:10 (2011), 3497-3512.

[28] J. C. Robinson, Infinite-dimensional dynamical systems. An introduction to dissipative parabolic PDEs and the theory of global attractors, Cambridge Texts Appl. Math., Cambridge Univ. Press, Cambridge, 2001, xviii+461 pp.

[29] J. C. Robinson, Dimensions, embeddings, and attractors, Cambridge Tracts in Math., 186, Cambridge Univ. Press, Cambridge, 2011, xii+205 pp.

[30] А.В. Романов, "Точные оценки размерности инерциальных многообразий для нелинейных параболических уравнений”, Изв. РАН. Сер. матем., 57:4 (1993), 36-54; англ. пер.: A. V. Romanov, "Sharp estimates of the dimension of inertial manifolds for nonlinear parabolic equations", Russian Acad. Sci. Izv. Math., 43:1 (1994), 31-47.

[31] А.В. Романов, “Три контрпримера в теории инерциальных многообразий”, Матем. заметки, 68:3 (2000), 439-447; англ. пер.: А. V. Romanov, "Three counterexamples in the theory of inertial manifolds", Math. Notes, 68:3 (2000), 378-385.

[32] А. В. Романов, "Конечномерная предельная динамика диссипативных параболических уравнений”, Матем. сб., 191:3 (2000), 99-112; англ. пер.: А. V. Romanov, "Finite-dimensional limiting dynamics for dissipative parabolic equations", Sb. Math., 191:3 (2000), 415-429.

[33] А. В. Романов, "Конечномерность динамики на аттракторе для нелинейных параболических уравнений”, Изв. РАН. Сер. матем., 65:5 (2001), 129-152; англ. пер.: A. V. Romanov, "Finite-dimensional dynamics on attractors of non-linear parabolic equations", Izv. Math., 65:5 (2001), 977-1001.

[34] A. Romanov, "Finite-dimensional limit dynamics of semilinear parabolic equations" (to appear).

[35] R. Temam, Infinite-dimensional dynamical systems in mechanics and physics, 2nd ed., Appl. Math. Sci., 68, Springer-Verlag, New York, 1997, xxii+648 pp.

\section{А. Еден (А. Eden)}

Bogazici University, Bebek, Istanbul, Turkey

Поступила в редакцию

E-mail: eden@boun.edu.tr

C. В. Зелик (S. V. Zelik)

University of Surrey, Guildford, UK

E-mail: S.Zelik@surrey.ac.uk

\section{В. К. Калантаров (V. K. Kalantarov)}

Koç University, Istanbul, Turkey

E-mail: vkalantarov@ku.edu.tr 